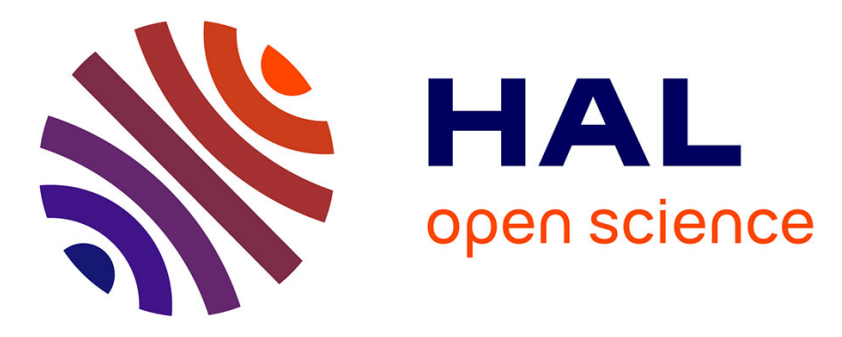

\title{
Analytical and 3D numerical analysis of the thermoviscoelastic behavior of concrete-like materials including interfaces
}

\author{
Benoît Bary, Christophe Bourcier, Thomas Helfer
}

\section{To cite this version:}

Benoît Bary, Christophe Bourcier, Thomas Helfer. Analytical and 3D numerical analysis of the thermoviscoelastic behavior of concrete-like materials including interfaces. Advances in Engineering Software, 2017, 112, pp.16-30. 10.1016/j.advengsoft.2017.06.006 · cea-02415668

HAL Id: cea-02415668 https://hal-cea.archives-ouvertes.fr/cea-02415668

Submitted on 18 Mar 2020

HAL is a multi-disciplinary open access archive for the deposit and dissemination of scientific research documents, whether they are published or not. The documents may come from teaching and research institutions in France or abroad, or from public or private research centers.
L'archive ouverte pluridisciplinaire HAL, est destinée au dépôt et à la diffusion de documents scientifiques de niveau recherche, publiés ou non, émanant des établissements d'enseignement et de recherche français ou étrangers, des laboratoires publics ou privés. 


\title{
Analytical and 3D numerical analysis of the thermoviscoelastic behavior of concrete-like materials including interfaces
}

\author{
Benoit Bary $^{1 *}$, Christophe Bourcier ${ }^{2}$, Thomas Helfer ${ }^{3}$ \\ ${ }^{1}$ Den-Service d’Etude du Comportement des Radionucléides (SECR), CEA, Université Paris- \\ Saclay, F-91191, Gif-sur-Yvette, France. e-mail: benoit.bary@cea.fr \\ ${ }^{2}$ Den-Service de Thermo-Hydraulique et de Mécanique des Fluides (STMF), CEA, Université \\ Paris-Saclay, F-91191 Gif/Yvette, France. e-mail: christophe.bourcier@cea.fr \\ ${ }^{3}$ Den-Service d'Etude et de Simulation du Comportement des Combustibles (SESC), CEA, \\ Bât. 151, 13108 St Paul lez Durance, France. e-mail: thomas.helfer@cea.fr
}

\begin{abstract}
:
We investigate in this paper analytically and numerically by means of $3 \mathrm{D}$ simulations the viscoelastic behavior of concrete and mortar subjected to creep loading and moderate temperatures at mesoscale. These heterogeneous materials are assumed to be composed of thermoelastic aggregates distributed in a linear thermoviscoelastic matrix; moreover, the Interfacial Transition Zones (ITZ) between aggregates and matrix, whose behavior is also considered as linear thermoviscoelastic, are explicitly introduced. The numerical specimens consist in unstructured periodic meshes containing polyhedral aggregates with various size and shapes randomly distributed in a box. Zero-thickness interface finite elements are introduced between aggregates and matrix to model the ITZ. Macroscopic response and averaged stresses and strains in the matrix and aggregate phases are compared to analytical estimations obtained with classical mean-field approximation schemes applied in the Laplace-Carson space, in which the ITZ are introduced via imperfect interfaces modelled with the Linear Spring Model (LSM). The effects of ITZ thickness, aggregate shape and uniform temperature increase are then studied to evaluate their respective influence on the local and macroscopic creep behavior of mortar and concrete. Globally, it is found that the model response is in relatively good agreement with numerical simulations results, and that as expected while the ITZ do not affect significantly the concrete behavior, they have a nonnegligible impact on the mortar one.
\end{abstract}

Keywords: 3D finite element simulations; concrete and mortar behavior; linear thermoviscoelasticity; analytical homogenization; Interfacial Transition Zone; imperfect interfaces.

${ }^{*}$ Corresponding author

Page 1 


\section{Introduction}

When considering concrete structures in the nuclear context (i.e. containment building, waste storage structures...), two main functions have generally to be guaranteed: containment and protection against radionuclide migration. The constitutive material must then meet high requirements in terms of performance and durability. In particular, loss of containment abilities due to long-term creep and induced development of cracks shall be prohibited. It is well known that concrete is a heterogeneous material made up at the mesoscale of linear elastic aggregates distributed in a mortar matrix whose behaviour is time-dependent. Besides, the presence of an ITZ (Interfacial Transition Zone) constituted by a thin interface between the aggregates and the matrix is known to also influence the overall behaviour, due to its lesser mechanical (and higher transport) properties, see e.g. [1-4]. It is then of particular importance to correctly characterize the respective role and impact of both phases and ITZ regarding the creep strains, since the initiation and propagation of cracks are strongly related to the local stresses and strains states as well as their history.

In the literature, while a number of numerical studies address the problem of the effects of ITZ influence in cementitious materials in elasticity (e.g. [5,6]), less are developed in a viscoelastic framework, at least partly due to the resulting more complex formulation. From the theoretical viewpoint, numerous approaches for estimating mechanical as well as transport properties of cementitious materials including ITZ are based on an explicit representation of the interface and the use of analytical homogenization techniques such as the self-consistent scheme to upscale the physical properties, see e.g. [1,7-10]. However, if such developments are relatively well adapted to the case where the interface thickness is moderately low (i.e. mortars), they appear by contrast less satisfactory for concretes since the ratio of respective size between the interfaces and aggregates ranges from 2 to 3 orders of magnitude. Models devoted to thin interfaces, i.e. accounting specially for the small size of the interface with respect to the inclusion one, are comparatively more suited to the case of ITZ surrounding aggregates and coarse sand particles. In particular, the resulting imperfect interfaces whose material properties are lower than the inclusion ones may be properly approached by the Linear Spring Model (LSM) developed by e.g. [11-13]. This model describes the displacement jump across the interface as a function of the normal stress (assuming the stress jump is zero) via rigidity coefficients estimated with simple expressions involving the interface mechanical properties and its thickness $h$. Such model has been successfully applied to the simulation of imperfect interfacial debonding [14] and thin and compliant interfaces (e.g. $[13,15])$.

Numerically, the study of interface effects in diverse situations has also generated interest among the research community (see e.g. $[6,16]$ ). The advantages of numerical simulations are that they 
allow considering in the material samples various and more complex configurations regarding aggregate shapes and sizes, including possible different ITZ thicknesses and mechanical properties, which cannot be reached in general analytically. Also, local analyses of physical fields (strain, stress, temperature, etc.) can be performed so as to quantify statistical information as dispersion in aggregates, ITZ and matrix subvolumes, contrary to analytical upscaling schemes which are mostly limited to per-phase averaged quantities. Diverse methods have been applied to solve the problem of heterogeneous materials including interfaces: among others, one can cite the classical finite element method (FEM) [17,18], lattice models [19-21], XFEM/level set methods $[22,23]$, and FFT-based methods [3]. We note that in some of these references, interfaces are explicitly introduced without the recourse of special elements or procedures, meaning that the results directly depend on the spatial resolution/discretization of the material. Some other methods make use of homogenized zones encompassing ITZ to avoid their explicit introduction. Interestingly, a number of studies have emphasized the interest of specific interface elements for describing fracture processes in addition to ITZ effects in cementitious materials via cohesivezone models, see e.g. [24-26].

In this paper we investigate analytically and numerically the thermoviscoelastic behavior of concrete and mortar at mesoscale, with a particular focus on the effects of ITZ. One purpose of the study is to test the capacities of simplified analytical models based on classical estimation methods against computation results performed on numerical samples expectedly (much) more representative of such concrete and mortar materials. Specifically, the simulations are carried out by applying the FEM on 3D specimens consisting in polyhedral aggregates of various sizes and shapes randomly distributed in a box. We make use of previous developments and procedures to generate mesoscale numerical samples and to perform computational analyses [27-30]. The matrix and ITZ phases are considered as linear thermoviscoelastic materials ruled by different generalized Maxwell models. Specific interface elements are introduced between the aggregates and the matrix to simulate the ITZ, whose behavior is characterized by the LSM extended to the thermoviscoelastic case. These interface elements are considered to be very well suited for this purpose, and are one good reason to carry out FEM simulations, though it should be noticed that alternative methods may be more efficient than FEM for general numerical simulations (i.e. without interfaces) of representative element volumes (REV), see e.g. [31]. Note that the aggregates in the placement procedure are not allowed to intersect each other by construction, so that possible ITZ percolation effects are not reproduced; this is also the case for the analytical model since the microstructure representation takes only into account separate inclusions. 
A particular attention will be put on the analysis of the overall and intra-phase response of the numerical specimens when subjected to classical creep and heating loadings. In particular, the evolution of averaged stresses and strains in the matrix and aggregate phases will be reported and compared to the analytical estimations obtained with different mean-field approximation schemes applied in the Laplace-Carson (LC) space. The influence of the ITZ thickness on the overall and local response of the specimens will also be studied. We expect recover the quantitative results highlighting the much more importance of ITZ in mortar behavior than in concrete one. Further, the impact of the aggregate shape on both local and macroscopic response will be analyzed through different mesostructures with flat and elongated particles. Finally, a local analysis regarding the evolution of the mean stresses and strains in each aggregate and in matrix subvolumes will be performed so as to quantify their dispersion.

\section{Linear viscoelastic modelling}

As mentioned in the introduction, we are concerned in this paper with the analysis of the thermoviscoelastic behavior of concrete with a special focus on the effects of the ITZ. In this section the models used for the viscoelastic formulation are recalled, while the extension to the thermoviscoelastic case is detailed in section 5 .

\subsection{Matrix behavior}

The behavior of the matrix material is assumed to be linear viscoelastic, with bulk $k^{m}(t)$ and shear $\mu^{m}(t)$ moduli ruled separately by a generalized Maxwell model with $N+1$ elements (see Figure 1 for the representation of a Maxwell model with the element labelled as 0 composed only of a spring) as:

$$
k^{m}(t)=k_{0}^{m}+\sum_{i=1}^{N} k_{i}^{m} e^{-t / \tau_{i}^{m}}, \mu^{m}(t)=\mu_{0}^{m}+\sum_{i=1}^{N} \mu_{i}^{m} e^{-{ }^{t} / \tau_{i}^{m}}
$$

in which $k_{i}^{m}$ and $\mu_{i}^{m}$ with $i \in\{0, N\}$ are the elastic moduli of the Maxwell elements, $\tau_{j}^{m}$ are their relaxation times, $N$ is the number of viscoelastic elements. It is assumed to simplify that $\tau_{j}^{m}$ and $N$ are the same for both moduli, but it is not strictly mandatory. Accordingly the behavior law takes the form:

$$
\boldsymbol{\sigma}_{m}(t)=3 \int_{0^{-}}^{t} k^{m}(t-\tau) \frac{d \epsilon_{m}}{d \tau} d \tau \mathbf{1}+2 \int_{0^{-}}^{t} \mu^{m}(t-\tau) \frac{d \boldsymbol{e}_{m}}{d \tau} d \tau
$$


with $\boldsymbol{\sigma}_{m}(t), \epsilon_{m}=1 / 3 \operatorname{tr}\left(\boldsymbol{\varepsilon}_{m}\right)$ and $\boldsymbol{e}_{m}$ the macroscopic stress tensor, bulk and deviatoric part of the strain tensor $\boldsymbol{\varepsilon}_{m}$ of the matrix, respectively; $\mathbf{1}$ is the second-order identity tensor..

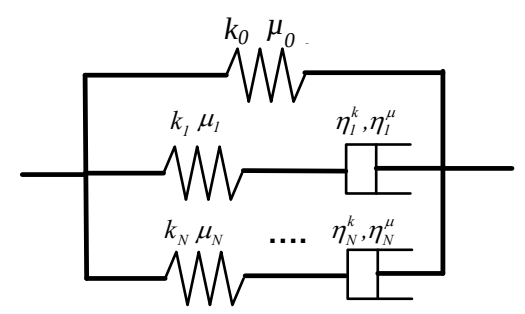

Figure 1. Representation of a Generalized Maxwell model with $\mathrm{N}+1$ elements.

It is well-known that in the case of linear viscoelasticity the time-dependent problem may be equivalently reformulated as a linear elastic problem in the Laplace-Carson (LC) space, allowing to applying classical upscaling techniques (see e.g. [32-35]). We recall that the LC transform $\tilde{f}(s)$ of a function $f(t)$ takes the following form:

$$
\tilde{f}(s)=s \int_{0}^{\infty} e^{-s t} f(t) d t
$$

with $s$ the variable in the LC space. One advantage of this transformation is to replace the time integral formulation (2) by an equivalent elastic-like formulation in the LC space as:

$$
\tilde{\boldsymbol{\sigma}}_{m}(s)=3 \tilde{k}^{m}(s) \tilde{\epsilon}_{m} \mathbf{1}+2 \tilde{\mu}^{m}(s) \tilde{\boldsymbol{e}}_{m}
$$

This procedure is referred to as correspondence principle. The expressions of $\tilde{k}^{m}(s)$ and $\tilde{\mu}^{m}(s)$ obtained from (3) take the following simple form:

$$
\tilde{k}^{m}(s)=k_{0}^{m}+\sum_{i=1}^{n} k_{i}^{m} \frac{s}{s+1 / \tau_{i}^{m}}, \tilde{\mu}^{m}(s)=\mu_{0}^{m}+\sum_{i=1}^{n} \mu_{i}^{m} \frac{s}{s+1 / \tau_{i}^{m}}
$$

As it is well known, one drawback with the LC transform technique is to obtain the inverse transform in the time space of the formulation expressed in the LC space. Diverse methods have been developed for this purpose, see e.g. [36,37]. Here we will make use of the classical numerical inversion technique known as collocation method, which proposes to approximate the functions as Prony (or Dirichlet) series, for they take a particularly simple and tractable form (analytically and numerically) in both LC and time space (see e.g. [38,39]). Interestingly, it is shown in [40] that in the particular case of the Mori-Tanaka (MT) scheme and spherical particles, exact or semianalytical solutions of the inversion problem can be derived, leading to expressions in the time space also in the form of Prony series. 
Whatever the method used to carry out the inversion of the LC transform, the macroscopic behavior may then take the form of a linear viscoelastic material:

$$
\boldsymbol{\sigma}(t)=3 \int_{0^{-}}^{t} k^{h o m}(t-\tau) \frac{d \epsilon}{d \tau} d \tau \mathbf{1}+2 \int_{0^{-}}^{t} \mu^{h o m}(t-\tau) \frac{d \boldsymbol{e}}{d \tau} d \tau
$$

where the same notations as in Eq. (2) has been used; $k^{\text {hom }}$ and $\mu^{\text {hom }}$ denote the homogenized bulk and shear moduli, which are then expressed in the form of Prony series as defined in Eq. (1) but with possibly a different number of viscoelastic chains and associated characteristic times for both moduli.

\subsection{Interface modelling}

As mentioned above, the behavior of the material composing the interfaces between the aggregates and the matrix is assumed to be ruled by generalized Maxwell models. The ITZ being very thin with respect to the aggregate size, we propose in this study to represent them by imperfect interfaces. Such approach is relatively simple and suits well with a finite element (FE) implementation with specific interface elements. In concrete and mortar, the mechanical properties of the ITZ are known to be more compliant than those of the matrix and aggregates; we then chose to apply the LSM in the LC space to simulate the interface effects. The interface conditions for the LSM in the LC space are (e.g., $[11,41,42])$ :

$$
[\tilde{\boldsymbol{\sigma}}] . \boldsymbol{n}=0, \tilde{\mathbf{k}} \cdot[\tilde{\boldsymbol{u}}]=\tilde{\boldsymbol{\sigma}} \cdot \boldsymbol{n}
$$

where $\boldsymbol{n}, \tilde{\mathbf{k}}, \tilde{\boldsymbol{u}}$ are the unit normal vector to the interface, the second order tensor characterizing the elastic parameters in the LC space, and the displacement (see Figure 2); [ $]$ denotes the jump of the corresponding quantities. Eq. (7) expresses that the normal stress (or traction) is continuous while the displacements exhibit a jump across the interface. For infinite values of the parameters, the interface displacement jumps vanish, leading to a perfect bonding interface, whereas zero values entail zero interface tractions and consequently debonding of matrix and inclusions. Intermediary positive values of these parameters then characterize an imperfect interface [12].

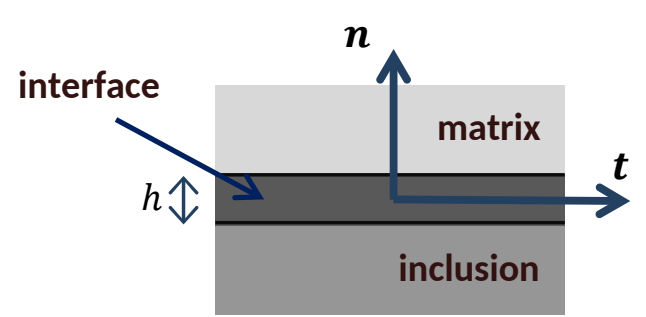

Figure 2. Schematic representation of a thin interface between an inclusion and the matrix. 
In the case where the interface is thin and compliant with respect to the inclusion:

$$
h \ll R_{i}, \tilde{E}_{c} \ll \tilde{E}_{i}, \tilde{\mu}_{c} \ll \tilde{\mu}_{i}
$$

with $h$ the thickness of the interface, $R_{i}$ the radius of the spherical inclusion, $\tilde{E}_{c}$ and $\tilde{\mu}_{c}$ the Young and shear moduli of the interface, $\tilde{E}_{i}$ and $\tilde{\mu}_{i}$ the Young and shear moduli of the inclusion, the tensor $\tilde{\mathbf{k}}$ can be expressed as $\tilde{\mathbf{k}}=\tilde{k}_{n} \boldsymbol{n} \otimes \boldsymbol{n}+\tilde{k}_{t} \boldsymbol{s} \otimes \boldsymbol{s}+\tilde{k}_{t} \boldsymbol{t} \otimes \boldsymbol{t}$ with:

$$
\tilde{k}_{n}=\frac{2 \tilde{\mu}_{c}\left(1-\tilde{v}_{c}\right)}{h\left(1-2 \tilde{v}_{c}\right)}, \tilde{k}_{t}=\frac{\tilde{\mu}_{c}}{h}
$$

in which $s$ and $\boldsymbol{t}$ are the two orthogonal unit vectors in the tangent plane of the interface and $\tilde{v}_{c}$ is the Poisson ratio of the interface. In the following, we will retain this model for characterizing the properties of the interfaces in both FE simulations and analytical models, providing $\tilde{k}_{n}$ and $\tilde{k}_{t}$ are expressed in the LC space.

In order to evaluate the response of the LSM in the particular case of ITZ where the conditions regarding the mechanical properties in Eq. (8) are only approximately filled, we also propose to apply a more sophisticated approach recently derived in [43]. In this approach, both interfacial displacement and traction discontinuities occur, i.e. Eqs. (7) are no more valid. This means in particular that the conditions involving the mechanical properties of the inclusion and interface in Eq. (8) need not to be satisfied. The model then includes the LSM as a special case. As its formulation is relatively complex and the obtained expressions lengthy, they are not recalled here and the reader is invited to refer to [43] for the details of the developments.

\subsection{Analytical homogenization with interfaces}

By construction, the heterogeneous materials considered in this study exhibit composite inclusions composed of the aggregates and their surrounding imperfect interfaces of zero thickness. Consequently the classical mean-field homogenization schemes cannot directly be applied, even in the LC space. We then propose to estimate the overall properties of the heterogeneous material by first making use and extending to the linear viscoelastic case the approach due to $[12,41]$, based on a replacement procedure. In this approach, the elastic spherical composite inclusions are replaced by energetically equivalent homogeneous spherical inclusions (see Figure 3) assumed to be perfectly bonded to the matrix. To be more precise, the procedure consists in substituting an infinite elastic matrix containing an imperfectly bonded spherical elastic inclusion of radius $R_{i}$ by the same infinite matrix comprising a perfectly bonded equivalent inclusion of radius $R_{i}$, providing that the elastic energy of both systems is equivalent. The obtained expressions of the moduli $\tilde{k}_{e q}$ and $\tilde{\mu}_{e q}$ for these equivalent particles depend then on both inclusion 
and interphase properties; they take the following form (see [41] for details about their formulation, which is beyond the scope of this paper):

$$
\begin{gathered}
\tilde{k}_{e q}=\frac{m_{r} \tilde{k}_{i} \tilde{\mu}_{m}}{3 \tilde{k}_{i}+m_{r} \tilde{\mu}_{m}} \\
\tilde{\mu}_{i}\left(24 M m_{\theta}+m_{r}\left(16 M+m_{\theta} N\right)\right) \\
80 g_{3} M+4 g_{3} m_{\theta}\left(10\left(7-\tilde{v}_{i}\right)+M\right)+m_{r}\left(2 g_{3}\left(140-80 \tilde{v}_{i}+3 M\right)+m_{\theta} N\right)
\end{gathered}
$$

with $M=g_{3}\left(7+5 \tilde{v}_{i}\right), \quad N=5\left(28-40 v_{i}+M\right), \quad g_{3}=\tilde{\mu}_{i} / \tilde{\mu}_{m}, \quad m_{r}=\tilde{k}_{n} R_{i} / \tilde{\mu}_{m}$ and $m_{\theta}=\tilde{k}_{t} R_{i} / \tilde{\mu}_{m}$. In these relations the indexes $m$ and $i$ relate to the matrix and inclusion phases, respectively. Since these formulas are established in an elastic framework, they are applied here in the LC space.

As mentioned in the previous subsection, a second approach in which the imperfect interfaces exhibit simultaneously interfacial displacement and traction discontinuities will also be applied [43]. In this reference, the same procedure as in $[12,41]$ is employed to replace the composite inclusions by an equivalent homogeneous one in an energetic sense. Again, as the expressions providing the moduli $\tilde{k}_{e q}$ and $\tilde{\mu}_{e q}$ of these equivalent spherical particles are very complicated and lengthy, they are not developed here (see [43] for details).

Once the composite inclusions are substituted by equivalent homogeneous ones, classical homogenization methods as the MT [44] or generalized self-consistent scheme (GSCS, [45,46]) may be applied to obtain the overall properties of the material. In the numerical applications, we will make use essentially of the GSCS which is known to give satisfying estimations even for relatively large inclusion volume fractions (see e.g. [12]). As this scheme is well-known, its formulation is not recalled here.

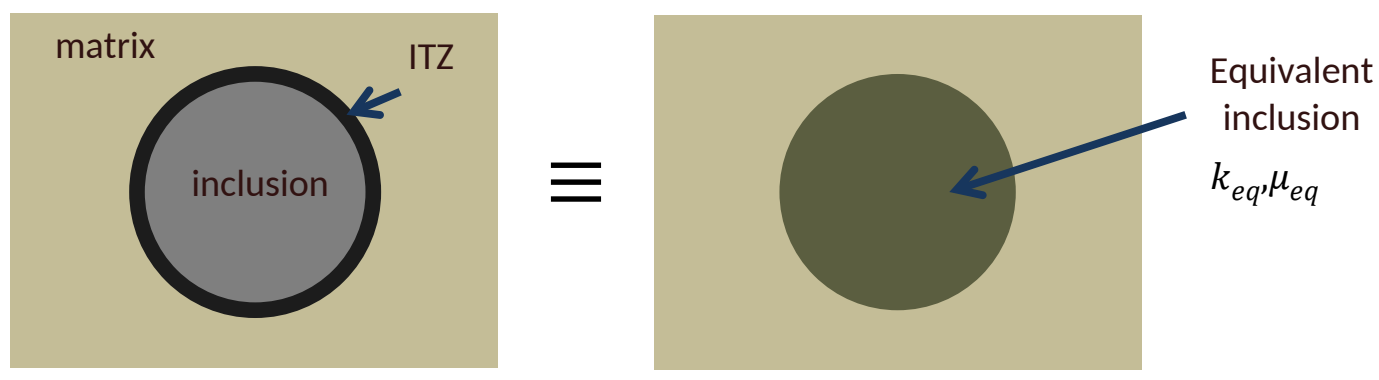

Figure 3. Description of the replacement procedure [41].

Note that in the alternative approach where the interfaces are modelled explicitly by a nonzero thickness phase surrounding the inclusions (Figure 3 left), the GSCS can be directly applied to the corresponding 3-phase microstructure. This scheme will be compared to the estimations obtained with the zero-thickness interface approach detailed above in section 4. 


\section{FE numerical applications}

\subsection{Mesostructure generation}

The procedure for constructing the numerical cubic samples is detailed in $[28,47]$. The open-source python library Combs [47] based on the Computer-Aided Design code Salome (http://www.salome -platform.org) is used to generate both geometry and meshes of the mesostructures. Basically, the geometries are obtained by randomly distributing in a box polyhedral aggregates of various sizes and shapes obtained independently by a classical Voronoi space decomposition [28]. Note that any types of aggregates may be considered in this process; for instance real aggregates obtained by tomography (see e.g. [48]) may also be introduced in the box. A minimal distance between two particles is prescribed by the user to avoid any interpenetration. As such, since the ITZ are modelled by zero-thickness interface elements, possible percolation effects are not considered. To improve the placement procedure time, the GJK 3D algorithm has been implemented in Combs for fast polyhedrons distance computation (see e.g. http://www.dyn4j.org/ 2010/04/gjk-distance-closest-points). The periodicity of the numerical samples is imposed so as to allow applying periodic boundary conditions. The automatic meshing softwares developed by Distene (http://www. meshgems.com/) are then used to generate the unstructured periodic meshes, with triangles for the surfaces (MeshGems-CADSurf) and tetrahedral elements for the volumes (MeshGems-Tetra). As mentioned in the introduction, this procedure has been applied in diverse situations for creating cementitious materials REVs or microstructural elements of concretes exhibiting a free surface exposed to the atmosphere [27-30].

In the following applications, we will consider two configurations of mesostructures, for describing concrete and mortar, respectively. They differ in fact only through their aggregate volume fraction. One reason is that it is not possible to generate samples with particles ranging from coarse aggregates to fine sand grains with a reasonable size in terms of number of elements and nodes. Then to limit the FE computation time and the placement procedure, the choice of 2 configurations with 872 and 4627 aggregates has been retained. These 2 configurations are assumed to represent concrete and mortar with a total volume fraction of 0.40 and 0.50 , respectively. The number and volume of the aggregates are defined to match approximately the ones of the corresponding spheres assembly according to the sieve curve described in [28]. The length edge of the samples is set to 120 and $24 \mathrm{~mm}$ for the concrete and mortar, respectively. Accordingly, the mean aggregate radius of an equivalent sphere assemblage is calculated as 3.55 and $1.05 \mathrm{~mm}$, respectively. Moreover, the samples with $40 \%$ of aggregates will also serve to analyze the effects of the boundary conditions (BC), see section 3.3.1. This particular analysis has indeed not been possible with the greater size meshes $(50 \%$ of aggregates) due to the memory limitation of the simulation PC-Linux machines available (currently 128 Go RAM, 
whereas 256 Go would be necessary to achieve these larger computations). The simulations are performed with the parallelized Cast $3 M$ FE code (www-cast3m.cea.fr) developed at CEA. Note that the total number of aggregates in the samples is systematically greater than the prescribed one since the aggregates overlapping the surfaces of the mesostructures are cut and the remaining parts are placed on the opposite faces to ensure periodicity of the geometry. To evaluate the effects of the aggregates shape, some mesostructures are generated with the initial Voronoi aggregates (denoted as 'isotropic' in the following), and others exhibiting flat and elongated inclusions. These flat and elongated aggregates are obtained by applying a scale transform of 3 to 4 on the initial aggregates along one or two perpendicular axes randomly defined.

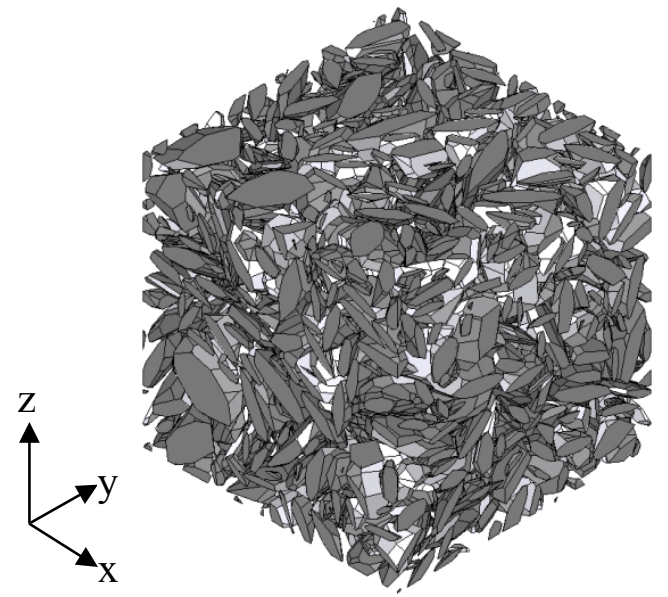

Flat, aspect ratio $=4$

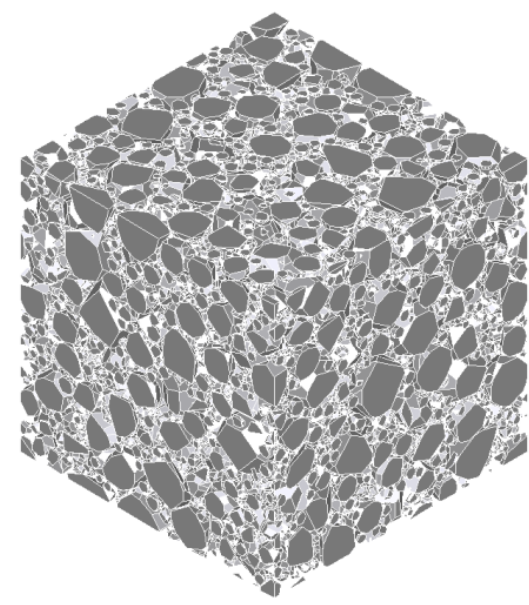

Isotropic

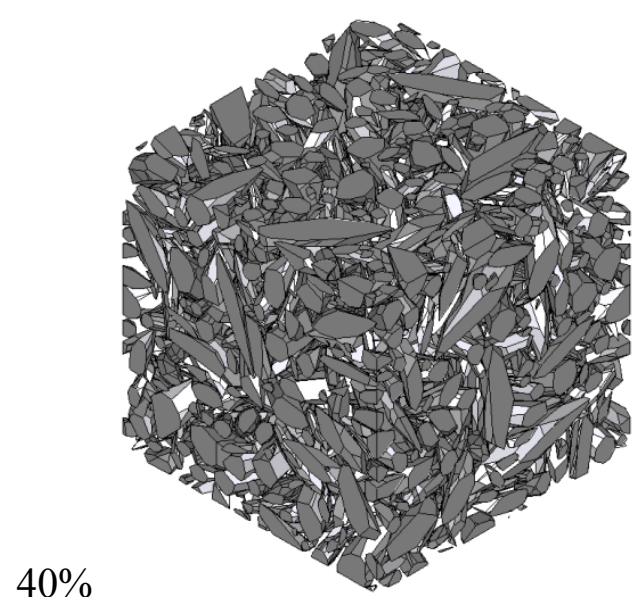

Elongated, aspect ratio $=4$

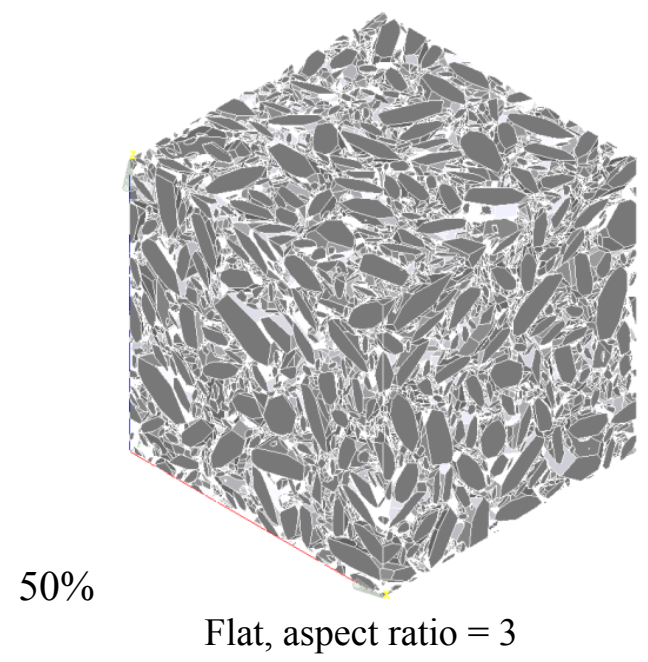

Figure 4. Sample realizations with $40 \%$ (top) and $50 \%$ (bottom) aggregate volume fraction, with isotropic, flat and elongated Voronoi particles.

As a consequence the volume fraction and corresponding number of aggregates are conserved for all mesostructures. Before being placed, the inclusions are rotated with a random angle around an 
axis also randomly defined. The Figure 4 presents sample realizations with the $40 \%$ (top) and 50\% (bottom) aggregates volume fraction, respectively, and with isotropic, flat, and elongated particles. A mesostructure with 872 spherical inclusions representing $40 \%$ of volume fraction (i.e. identical to the corresponding Voronoi aggregate cases) is also generated for comparison.

For the numerical results to be comparable, the meshes are constrained to have a similar size for each of the two volume fractions considered. Here the number of linear tetrahedral finite elements ranges from 2.23 to $2.37 \times 10^{6}$ and 3.71 to $4.21 \times 10^{6}$, for the $40 \%$ and $50 \%$ volume fraction mesostructures, respectively. The element size is controlled in the automatic meshing procedure by imposing globally the maximum length of the sides of the triangles composing all surface elements, i.e. interfaces and sample boundaries; it is chosen as 0.02 times the sample edge length. Moreover a 'gradation parameter' allows prescribing the maximum ratio between the lengths of the sides of two adjacent surface elements (triangles), set here to 1.3.

\subsection{Parameters}

The material parameters of both matrix and aggregates are taken the same as in [30]. The Young modulus of the aggregates is set to $E=70 \mathrm{GPa}$ and its Poisson ratio to $v=0.3$. The parameters of the Maxwell models are identified on a classical concrete creep test [49], assuming that the material is composed of the linear viscoelastic matrix in which are distributed $65 \%$ of spherical aggregate (no ITZ are considered), and applying the MT scheme to the resulting two-phase composite. The number of viscoelastic elements in the Maxwell models is set to $N=3$ in Eq. (1), and the relaxation times are chosen in accordance with the duration of the creep tests (300 days). The parameters characterizing these models are listed in Table 1. It should be noted that to simplify, the parameters of the matrix phase are kept the same for the two configurations, i.e. mortar and concrete, although they are in reality different. Without relevant experimental data on mortar, we have indeed considered somewhat artificial to perform an identification procedure for the mortar using the numerical non-consolidated data obtained from concrete matrix identification. Moreover, we believe that this choice does not affect the main features regarding analytical vs computational comparison results which are the focus of this study.

Table 1: Maxwell models parameters characterizing the matrix phase

\begin{tabular}{lllll}
\hline Element & $\mathbf{0}$ & $\mathbf{1}$ & $\mathbf{2}$ & $\mathbf{3}$ \\
\hline $\boldsymbol{k}_{\boldsymbol{i}}^{\boldsymbol{m}}(\mathbf{G P a})$ & 6.27 & 2.93 & 4.21 & 6.93 \\
$\boldsymbol{\mu}_{\boldsymbol{i}}^{\boldsymbol{m}}$ (GPa) & 3.41 & 7.77 & 3.54 & 3.32 \\
$\boldsymbol{\tau}_{\boldsymbol{i}}^{\boldsymbol{m}}$ (days) & & 2 & 20 & 150 \\
\hline
\end{tabular}


The ITZ mechanical properties have been estimated from the ones of the matrix by assuming that it is basically composed of this matrix phase, in which an additional porosity is incorporated. With the hypothesis that these supplementary pores are spherical, the MT scheme is then applied to obtain the homogenized properties of the corresponding interphase. The Figure 5 shows the time evolution of both bulk $k_{c}$ and shear $\mu_{c}$ moduli of the ITZ for several values of additional porosities $\phi_{a d}$ ranging from 0 to $40 \%$ of volume fraction. We observe as expected that the moduli are lower for higher porosities. In the following we adopt the value of $\phi_{a d}=30 \%$, which is a value close to the one commonly reported in the literature, see e.g. [1,2]. Note that this value $\phi_{a d}=30 \%$ provides moduli of about half the ones of the matrix phase, as reported in e.g. [50]. With such parameters, the behavior of the ITZ complies with the condition of applicability of the LSM defined in Eqs. (9), i.e. its mechanical properties are lower than the ones of the inclusions. The simplification regarding the fact that the matrix behavior is assumed the same for the $40 \%$ and $50 \%$ aggregate configurations implies that the ITZ properties are also the same in the two cases.
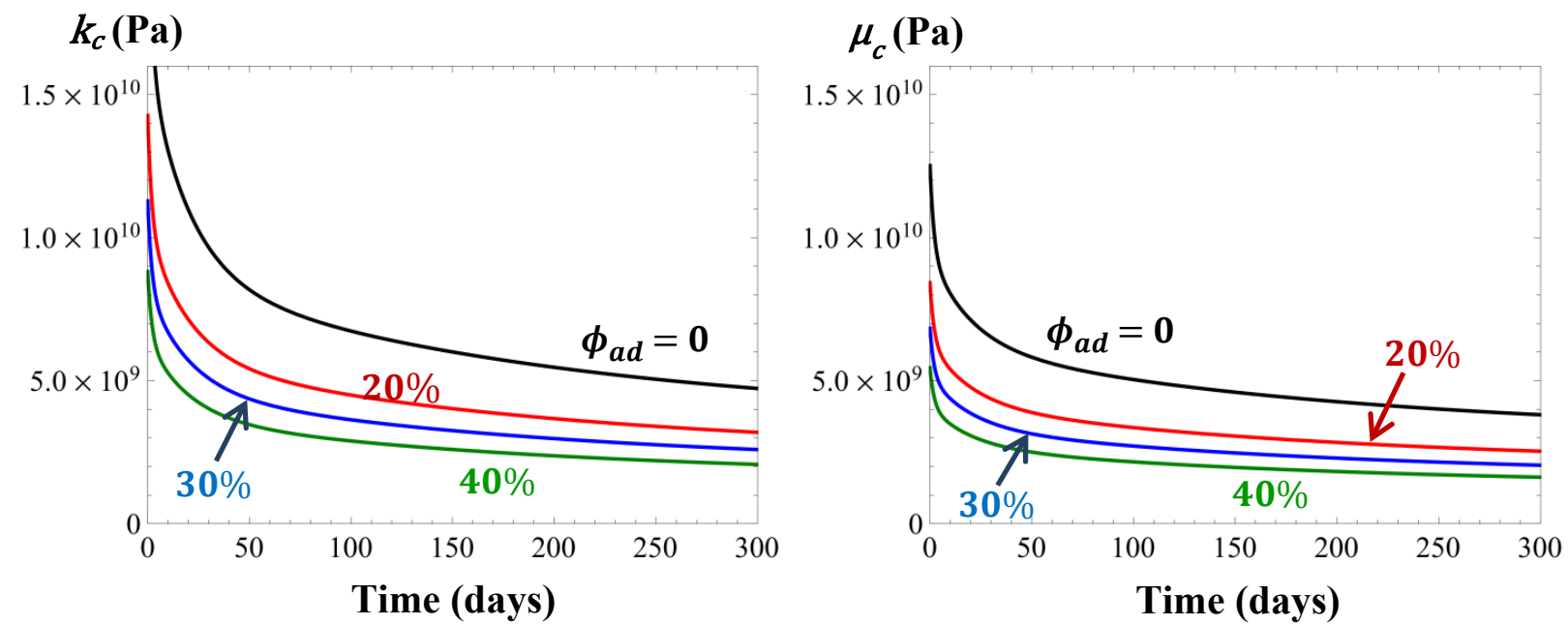

Figure 5. Time evolution of the bulk (left) and shear (right) moduli of the phase composing the ITZ, for different additional porosity volume fraction $\phi_{a d}$.

\subsection{Results with the $\mathbf{4 0} \%$ aggregate mesostructures}

We present in this section the results obtained in the case of the $40 \%$ aggregate volume fraction mesostructure. The main objectives are to analyze the effects of the $\mathrm{BC}$ and of the aggregate shape on the simulation results, in comparison with the analytical approach. To illustrate the potential role of the interfaces in the results, the different following values of interface thickness are considered $h=0.2,2,5,10$ and $20 \mu \mathrm{m}$. A calculation with no interfaces between aggregates and matrix is also carried out to control the relevancy of the results with smaller thicknesses. The loading corresponds to a typical creep test, defined by a constant normal stress of $-25.8 \times 10^{6} \mathrm{~Pa}$ 
applied on the sample surfaces in the direction $\mathbf{x}$ and zero stress in the other directions (see Figure 4). The computations are performed on a standard Linux machine with 20 cores and 128 Go RAM. The total simulated time is 300 days, reached in 32 time steps. The mechanical models for both matrix and interface materials have been implemented via the MFront code generator (www.tfel.sourceforge.net) developed at CEA [51]. Briefly speaking, MFront provides a set of domain specific languages for handling mechanical behaviors, which are meant to be easy to use and learn by researchers and engineers. A particular attention is paid to the robustness, reliability and numerical efficiency of the generated code. Moreover, MFront interface allows to generate code to be plugged in various software environments (languages or solver).

\subsubsection{Effects of boundary conditions}

We focus in this subsection on the particular effects of the BC on the simulation results, which are known to affect them depending in particular on the RVE size and the phase contrast, see e.g. [31,52]. Three classical types of boundary conditions are considered in the simulations: homogeneous strains KUBC and homogeneous stresses SUBC, which provide bounds for the isotropic moduli of numerical samples in elasticity (see e.g. [52-54]), and periodic PBC. The creep loading corresponds to homogeneous macroscopic stresses imposed at the sample surfaces, i.e. SUBC are directly applicable. KUBC are obtained by imposing to the nodes belonging to the sample surfaces displacements conforming to a homogeneous macroscopic strain (i.e., all nodes belonging to a face are constrained to have the same displacements), while the external load is prescribed to be the creep stresses. Likewise, PBC are set through constraints imposed between the nodes located on the exact opposite positions on the sample faces: the displacements of $\mathbf{2}$ face-to-face nodes is enforced to be the same. Obviously, such periodic $\mathrm{BC}$ are possible due to the periodic characteristics of the generated meshes. A consequence is that the aggregates crossing a sample face are mechanically connected to their remaining part intersecting the opposite face. One drawback of these $\mathrm{BC}$ involving supplementary displacement constraints is that they increase significantly the size of the numerical problem to solve.

The effects of these different $\mathrm{BC}$ are assessed by performing simulations on the same isotropic aggregate mesostructure with the creep loading defined above, and for various interface thicknesses. The results showing the calculated longitudinal creep strains up to 300 days for the values of $h=0.2$ and $20 \mu \mathrm{m}$ and the 3 considered BC are gathered on Figure 6 and Figure 7 for isotropic Voronoi aggregates and spherical aggregates mesostructures, respectively. On these Figures are also reported the analytical curves (plain lines) obtained by applying the procedure described in section 2.3 with the GSCS, then considering spherical inclusions. We observe 
significant differences whatever the interface thickness and the aggregates shape, with a slightly more discrepancy for the higher value of $h$. This confirms that the mesostructures we are dealing with cannot exactly be termed as representative elementary volumes. We observe as expected that the analytical curves are in better accordance with the results of the spherical aggregate case than with the Voronoi aggregate one. For the former configuration, the discrepancies are of about 3.5\% at 300 days between the analytical and PBC results for the two interface thicknesses, indicating a relatively good estimation of the model.
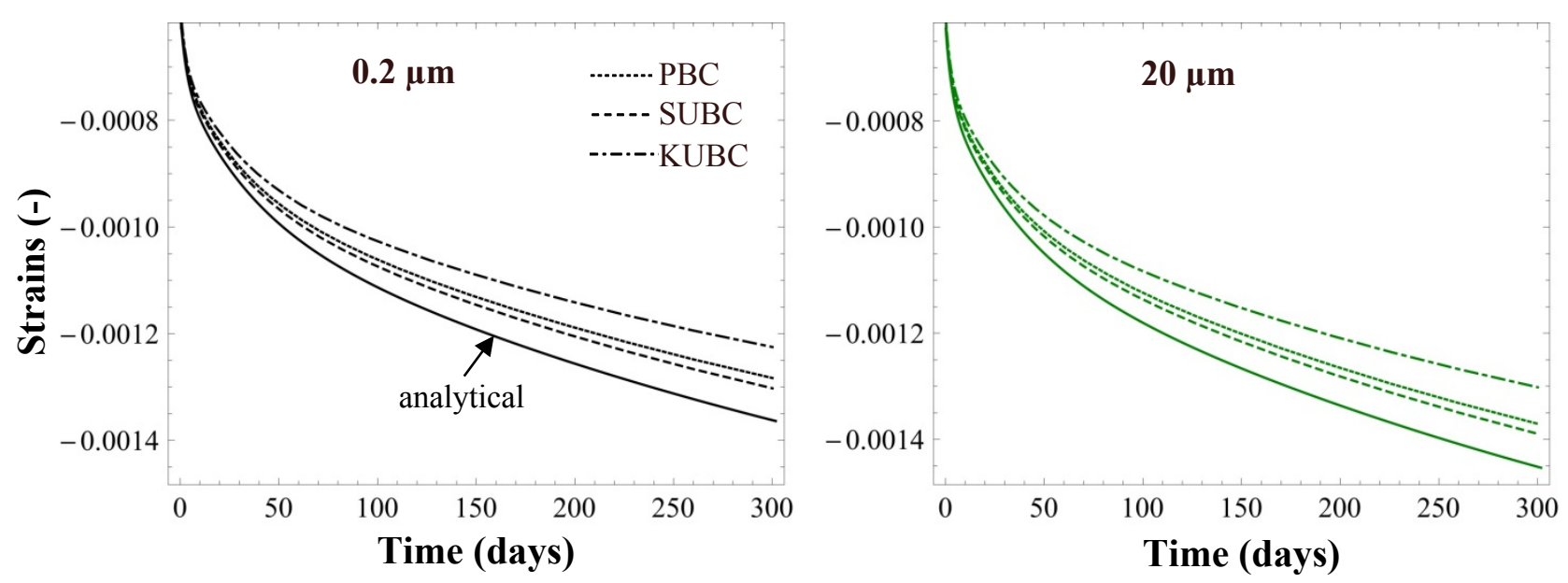

Figure 6. Analytical (GSCS) and numerical macroscopic longitudinal strain for interface thickness of 0.2 (left) and $20 \mu \mathrm{m}$ (right) obtained for different BC types in the case of the $40 \%$ isotropic Voronoi aggregate mesostructure.
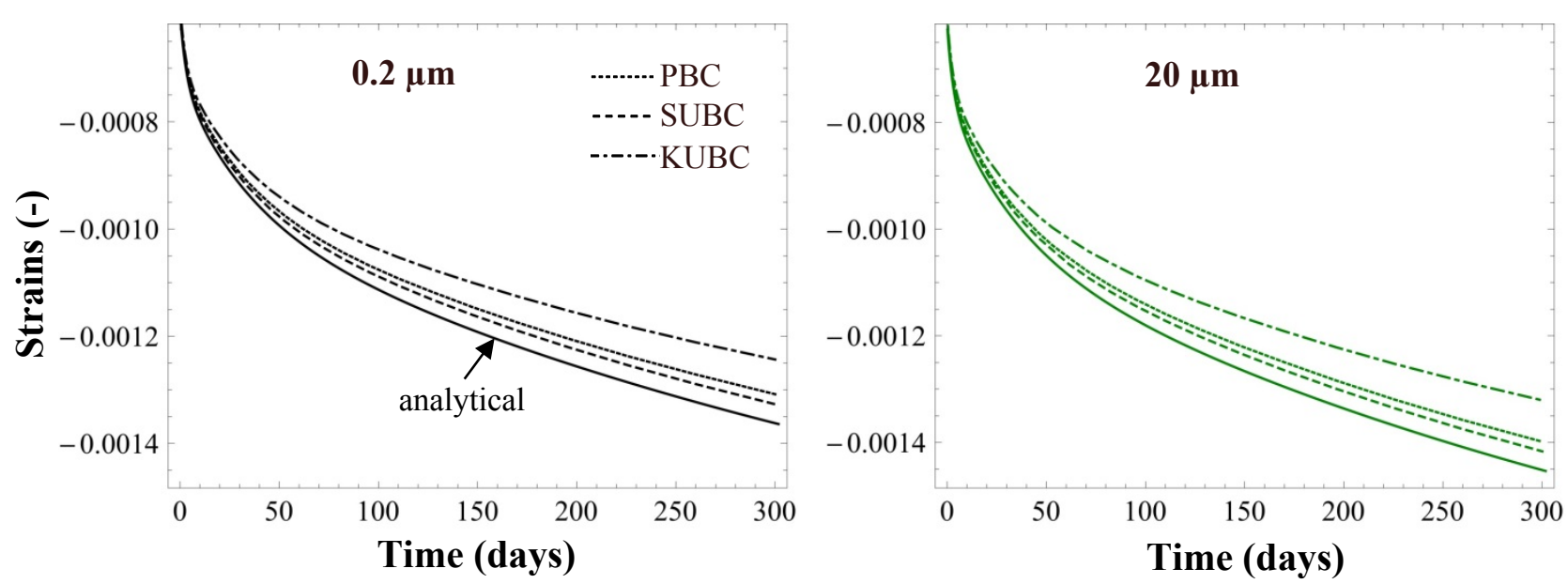

Figure 7. Analytical (GSCS) and numerical macroscopic longitudinal strain for interface thickness of 0.2 (left) and $20 \mu \mathrm{m}$ (right) obtained for different $\mathrm{BC}$ types in the case of the $40 \%$ spherical aggregate mesostructure.

Interestingly, we also note that the PBC results are much closer to the SUBC than to the KUBC ones; this agrees with the properties reported in particular in elasticity that in the case of a matrix more compliant than the inclusion phase (as in our case), SUBC results are closer than KUBC ones 
to the PBC simulations, which are generally considered as a good compromise and approach the best the 'real' results (see e.g. [55]). In the following, we will then essentially present simulations obtained with SUBC, as the computation time is much lesser than PBC due to the fact that the periodic $\mathrm{BC}$ are introduced in the FE models via supplementary constraints on the displacements of all nodes of the sample boundaries, which significantly increases the problem size to be solved. The simulations with SUBC are achieved in about 5-6 hours for the 40\% aggregate volume fraction cases, and additional 5-6 hours are needed for post-processing the results (i.e. calculating the average stresses and strains in all inclusions and matrix subvolumes for all time steps), as the corresponding processes are not parallelized.

\subsubsection{Effects of the aggregate shape}

We analyze in this subsection the particular effects of the aggregate shape on the numerical results. As mentioned, the considered aggregate shapes are spheres, isotropic Voronoi aggregates, and elongated and flattened aggregates with aspect ratio of 4 . All simulation results presented are carried out with SUBC, and are compared with analytical results estimated with the GSCS (3phase model) as explained in section 2.3. Figure 8 shows the macroscopic longitudinal creep strains calculated with interface thicknesses $h=0.2$ (left) and 20 (right) $\mu \mathrm{m}$. We observe that the magnitude of the creep strains is overestimated by the analytical model in both cases. The aggregate shape giving results the closest to the analytical curve is the sphere, followed by the isotropic Voronoi aggregate and the elongated and flat one. Note that the curves for these two modified Voronoi aggregate shapes are undistinguishable. We can then conclude that the aggregate shape has a relatively limited, though non-negligible, influence on the macroscopic response of the material: at 300 days the maximum deviation reaches about 5.5\%. As in elasticity, prolate and oblate shapes have more effects than spherical shape, i.e. in our case since the aggregates are more rigid than the matrix the overall behavior is more compliant with spherical inclusions; isotropic Voronoi aggregates influence may be seen as intermediary between spherical and flat/elongated aggregates.

Figure 9 shows the average strains (left) and stresses (right) in both matrix and inclusion phases for an interface thickness of $20 \mu \mathrm{m}$ and the different aggregate shapes. We note that the GSCS estimations are in relatively good agreement with the numerical results of strains, and by contrast the differences are more significant regarding stresses $(10 \%$ between spherical inclusions case and analytical curve). Again, as expected due to the spherical assumption in the modelling derivation the $\mathbf{F E}$ results with spheres are the closest to the model, followed by the isotropic then flat/elongated aggregates. It should be noted that the average strains in the aggregate phase cannot 
be directly obtained via the estimation of the strain concentration tensor. This is because in the approach of [41], the GSCS is applied to the material exhibiting equivalent homogeneous inclusions which replace the initial composite sphere + interface ones (see Figure 3). Consequently, the inclusive phase in the upscaling scheme relates to the composite spheres. One way to obtain the strains in the aggregate phase alone relies on the calculation of the stresses in the composite inclusions, which are the same than in the aggregates due to the stress continuity condition through the interfaces in Eq. (7), and from which the strain can be easily deduced due to the elastic behavior of the aggregates.
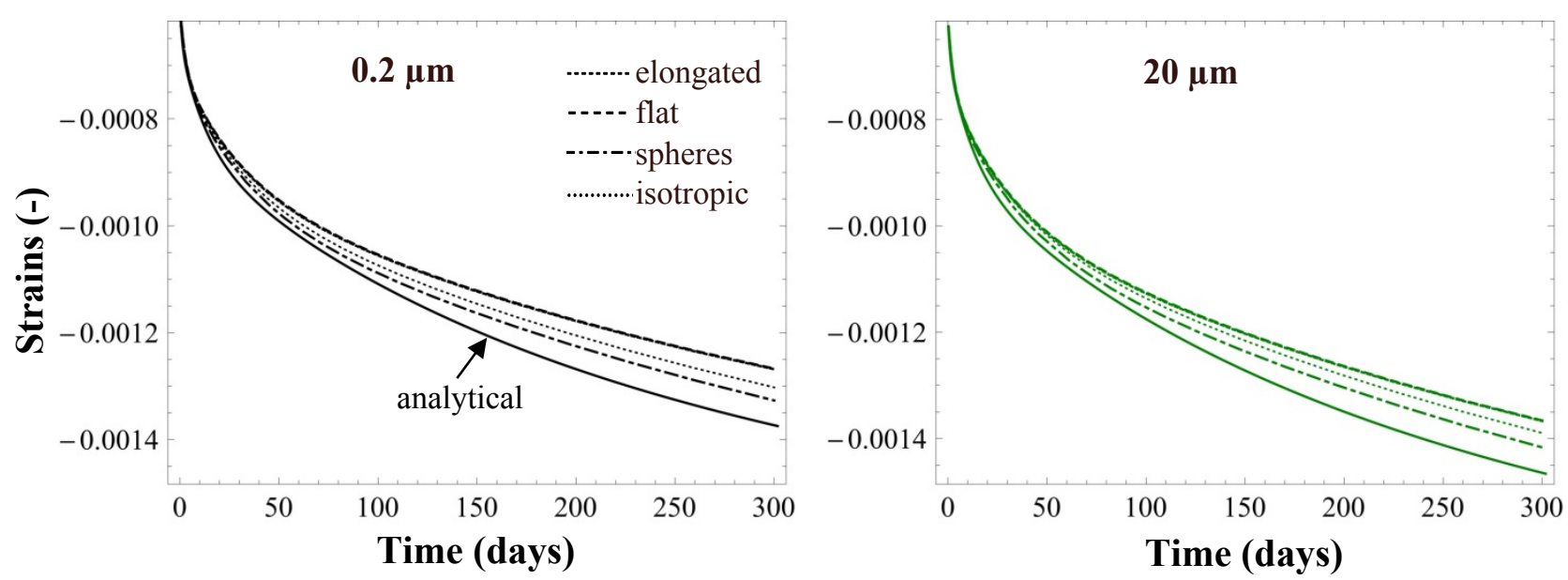

Figure 8. Analytical (GSCS) and numerical macroscopic longitudinal strain for interface thickness of 0.2 (left) and $20 \mu \mathrm{m}$ (right) obtained for different aggregate shapes in the case of the $40 \%$ aggregate mesostructure. The numerical results for the elongated and flat aggregates are almost superposed.
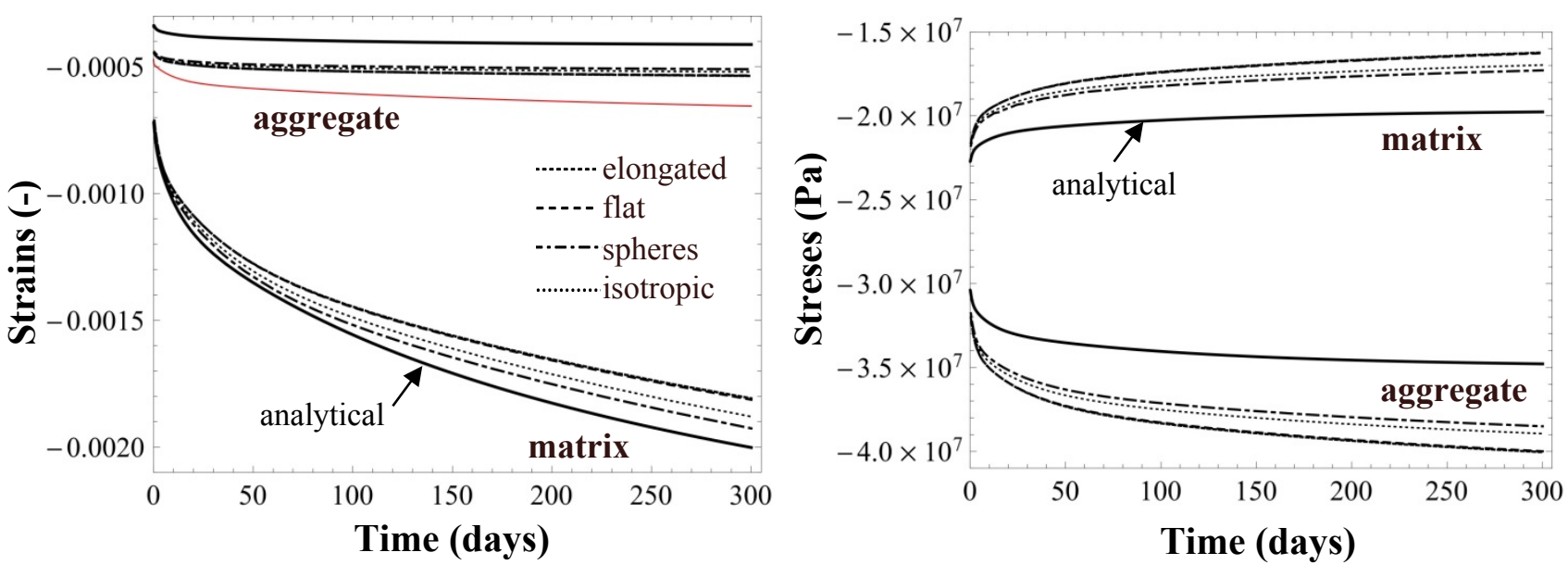

Figure 9. Analytical (GSCS) and numerical longitudinal strain (left) and stress (right) in both matrix and inclusion phases for interface thickness of $20 \mu \mathrm{m}$ obtained for different aggregate shapes in the case of the $40 \%$ aggregate mesostructure. The red plain curve on the left corresponds to the analytical strain of the composite aggregate (i.e. aggregate + interface). The numerical results for the elongated and flat aggregates are almost superposed. 
On Figure 9 left is reported the corresponding analytical curve in continuous black line, and in continuous red line the curve of the composite inclusions. We then observe that the numerical results lie nearly between the two analytical curves.

\section{Simulation results with the $50 \%$ aggregate mesostructures}

We present in this section the results obtained with the mesostructures with $50 \%$ of Voronoi aggregates. We focus our analysis on the effects of interface thicknesses, the comparison with different analytical homogenization schemes and the local behaviour, as the aspects regarding the effects of the aggregate shape and the $\mathrm{BC}$ have been discussed in section 3.3.

\subsection{Comparison with different analytical schemes}

We first study the effects of the different interface thicknesses of $h=1,25,50,100$ and $250 \mu \mathrm{m}$ on the 50\% aggregate mesostructures. These thicknesses are not representative of real values for concrete, and are chosen to clearly distinguish between the obtained curves, and also because by applying a size scale analogy (i.e. by dividing all sizes by 5 ) the results can be extended for mortars. Clearly speaking, the $\mathbf{4 0 \%}$ and $\mathbf{5 0 \%}$ samples can be used for both materials, providing an adjustment of the ITZ thickness to reflect a realistic ratio of ITZ over aggregate dimensions. Figure 10 presents the evolution of the creep longitudinal and transversal macroscopic strains obtained numerically by FE (symbols) and analytically (lines) for the isotropic aggregate case. The analytical results are obtained with the GSCS applied to the composite particles immersed in the matrix phase as depicted on Figure 3 right.

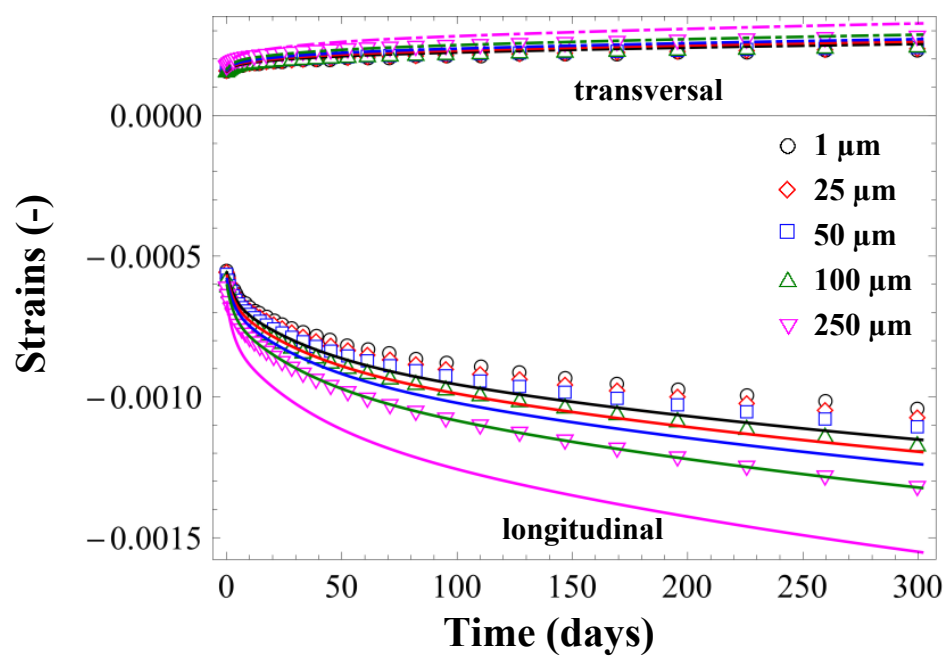

Figure 10. Longitudinal and transversal macroscopic creep strains obtained numerically (symbols) and analytically with the GSCS (lines) in the case of the $50 \%$ Voronoi aggregate for interface thicknesses of 1 to $250 \mu \mathrm{m}$. 
We observe as expected that the magnitude of strains progressively increases for higher values of thickness. The differences are small between $h=1$ and $25 \mu \mathrm{m}$, and are about 6,14 and $33 \%$ between $h=1$ and 50, 100 and $250 \mu \mathrm{m}$, respectively. Note that the results with no interface are omitted as they are superposed with the $h=1$ thickness ones. As already mentioned in the previous section, we may conclude that the presence of ITZ, whose typical thickness is around 20-25 $\mu \mathrm{m}$ [2] in concrete at mesoscale (i.e. considering the matrix as mortar), is not significant regarding the estimation of macroscopic response of the material. By contrast, for mortars which correspond approximately to the case of a thickness of $100 \mu \mathrm{m}$ with respect to the aggregate sizes, they appear very influential and should not be neglected. We also observe that the strains estimated by the model overestimate in magnitude the numerical ones for all interface thicknesses. Several causes may explain these discrepancies. The first and most important one, already discussed in the preceding section, lies in the difference between inclusion shape, spherical for the model and polyhedral in the simulations. Another is due to the inherent use of the GSCS whose estimations inevitably present some divergence from exact results, especially for important inclusion volume fractions. Biases introduced by the insufficiently fine meshes and the considered BC are also wellknown sources of deviation.
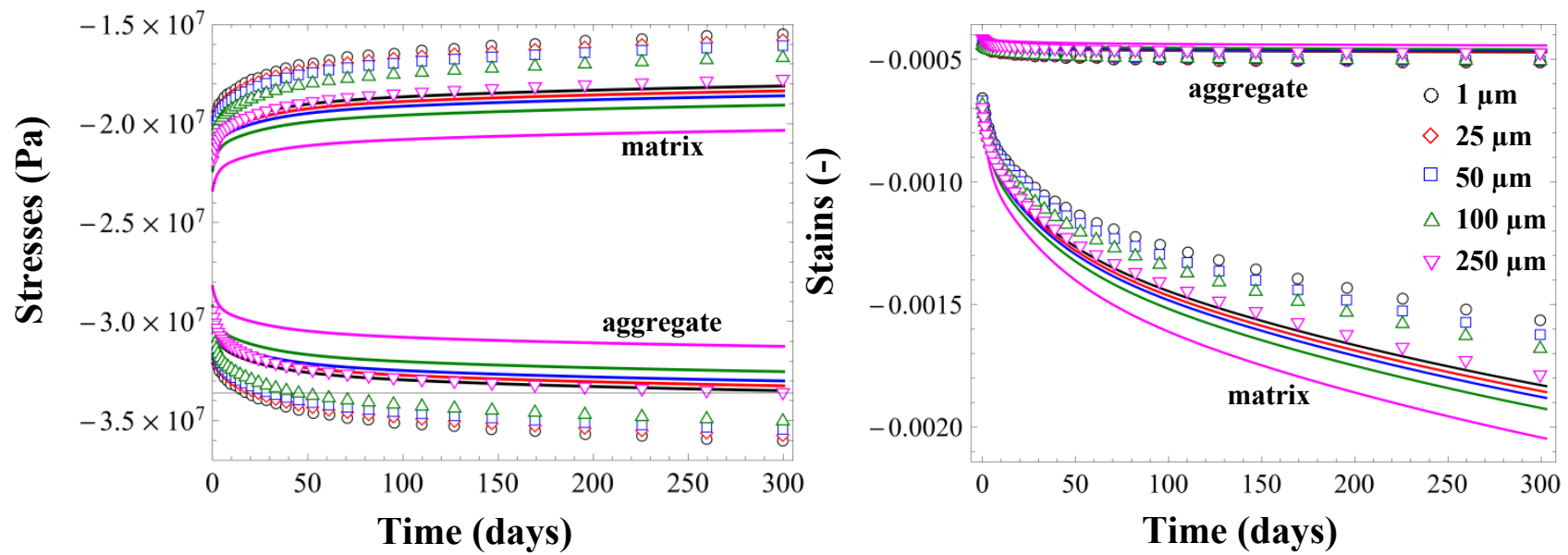

Figure 11. Average stresses (left) and strains (right) in both aggregate and matrix phase, obtained numerically (symbols) and analytically (lines) for different interface thicknesses ranging from 1 to $250 \mu \mathrm{m}$.

Figure 11 shows the evolution of average stresses (left) and strains (right) in the aggregate and matrix phases in the direction of creep loading, obtained numerically (symbols) and analytically (GSCS, lines), for the different interface thicknesses. This Figure, whose results are comparable to those of Figure 9 at a given interface thickness in the previous section, indicates that an increase of this thickness tends to reduce the magnitude of the stress in the aggregates and to augment it in the matrix. This is the same for the strain: it increases significantly in the matrix, and decreases in 
the aggregates. The range of variation is about $10 \%-12 \%$ for all cases between $h=1$ and $250 \mu \mathrm{m}$ at 300 days. Again, we consider that the model based on GSCS estimations provides results relatively close to the FE simulations.

To illustrate the response of the homogenization methods, Figure 12 presents the evolution of longitudinal creep strains obtained numerically (symbols) and analytically with different estimation schemes, for interface thicknesses of 1 (black curves), 100 (green curves) and 250 (pink curves) $\mu \mathrm{m}$. The continuous lines depict the analytical results provided by the GSCS associated with the replacement procedure and the LSM as defined in section 2.3 and shown in Figure 10. This case is labelled 'GSCS2 + LSM', GSCS2 referring to the fact that the GSCS is applied to a 2-phase composite. The dashed lines termed as 'GSCS2 + IDSD' result from the same application of both the GSCS and replacement procedure (i.e. to a 2-phase heterogeneous material), but the interface is modelled by the approach developed in [43]. As mentioned previously, in this approach both interfacial displacement and normal stress vector are allowed to be discontinuous across the thin interfaces. The dot-dash lines labeled 'GSCS3' give the evolution of the longitudinal strains from the GSCS applied to the heterogeneous material in which the ITZ is explicitly represented as a layer coating the spherical inclusion, see e.g. [7,56] for similar material description. In this case, the GSCS hypothesis on the composite sphere morphology makes the thickness of the layer varying with the inclusion size. For the applications, the volume fractions of the ITZ layer are calculated from the composite sphere with core size given by the mean aggregate size of the numerical model, equal here to $3.55 \mathrm{~mm}$. Last, the dotted lines labelled 'GSCS2 mult' correspond to an extension of the GSCS to multiphase composites as proposed in [57], and used for instance in [41]. This method is based on the following approximate decoupled formulation for $N$ particulate phases:

$$
\frac{\tilde{k}_{e q}}{\tilde{k}_{m}}=\prod_{i=1}^{N} \frac{\bar{k}_{i}}{\tilde{k}_{m}}\left(\phi_{i}, \tilde{k}_{i}, \tilde{k}_{m}, \tilde{\mu}_{i}, \tilde{\mu}_{m}\right), \frac{\tilde{\mu}_{e q}}{\tilde{\mu}_{m}}=\prod_{i=1}^{N} \frac{\bar{\mu}_{i}}{\tilde{\tilde{\mu}}_{m}}\left(\phi_{i}, \tilde{k}_{i}, \tilde{k}_{m}, \tilde{\mu}_{i}, \tilde{\mu}_{m}\right)
$$

where $\bar{k}_{i}$ and $\bar{\mu}_{i}$ are the moduli of a composite (estimated with the GSCS associated with the replacement procedure) containing only the $\mathrm{i}^{\text {th }}$ phase with volume fraction $\phi_{i}$ and moduli $\tilde{k}_{i}$ and $\tilde{\mu}_{i}$. Here, these relations are applied in order to circumvent the GSCS limitations and obtain a more realistic morphological representation of the dimensions of the ITZ with respect to the size of the aggregates. The aggregates are then divided in three classes with the following average sizes of inclusions: $8.8,5.89$ and $2.26 \mathrm{~mm}$, corresponding to a (total) volume fraction of $0.195,0.189$ and 0.116 , respectively. Since the ITZ thickness is constant, the theoretical volume fraction of the ITZ layer considered in the estimations differs in the three classes. 
We observe on Figure 12 that the estimations for $250 \mu \mathrm{m}$ provided by the GSCS2 + IDSD are relatively close to the ones given by the GSCS2 + LSM, indicating that the latter more simple model could be used with a correct accuracy in the context of a replacement procedure. On the other hand, the curves obtained with the GSCS with 3 phases, i.e. accounting explicitly for the ITZ and disregarding the replacement process, are more distant from the two other cases, and closer to the FE results. This means that the effects of the interfaces seem to be significantly lower in the GSCS3 case since the magnitude of the strains is lesser. We also note that the curves given by these 3 estimation methods are as expected undistinguishable for the thickness of $1 \mu \mathrm{m}$. Finally, the results obtained with the 'GSCS2 mult' procedure are nearer to the FE results than the 3 others for the higher ITZ thickness, and by contrast more distant for the lower one. Consequently the effects of the ITZ are weaker with this method since the difference of magnitude of the strain between 1 and $250 \mu \mathrm{m}$ is lower, which does not agree very well with the numerical data. For this reason its application is more questionable and may not be recommended here.
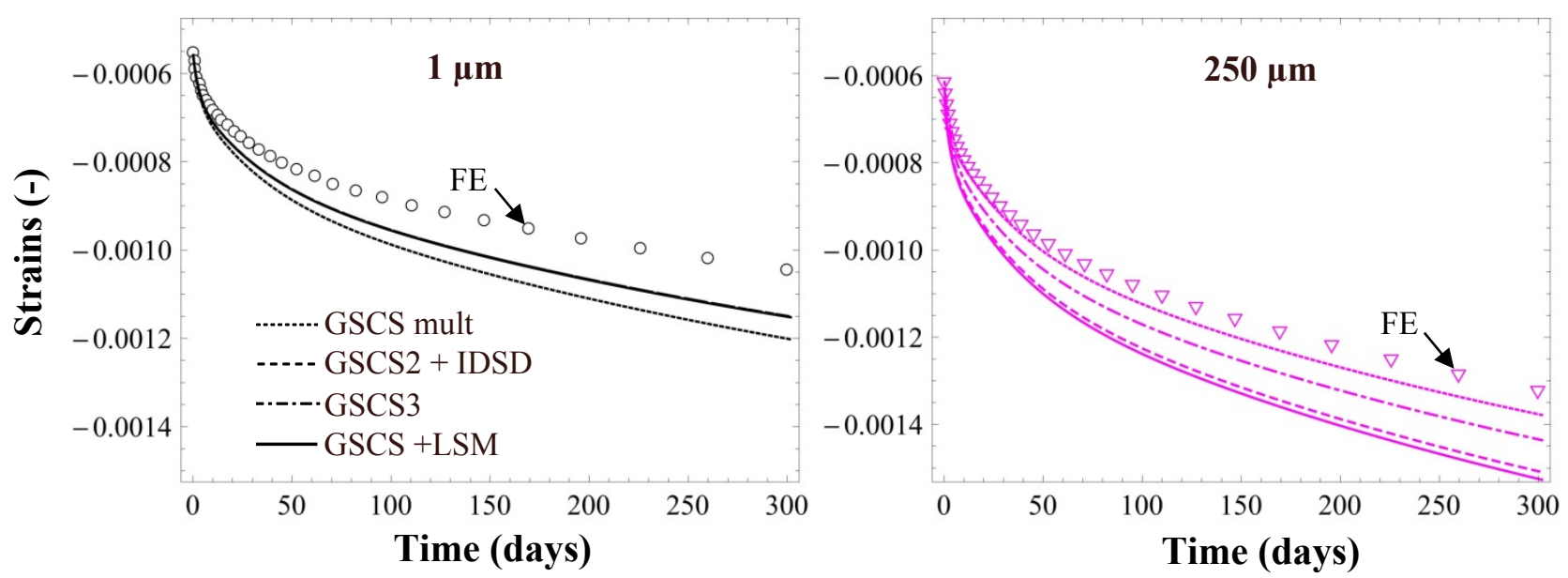

Figure 12. Longitudinal creep strains obtained numerically (symbols) and analytically with the GSCS2 + LSM (continuous lines), the GSCS2 + IDSD (dashed lines), the GSCS2 mul (dotted lines) and the GSCS3 (dotdash lines) for interface thicknesses of 1 (black, left) and 250 (pink, right) $\mu \mathrm{m}$.

To show the influence of the ITZ mechanical properties, Figure 13 depicts the FE longitudinal creep strain of the material for 3 different ITZ behaviors and thicknesses. The behaviors correspond to the values of additional porosities of 20,30 and 40\% in the ITZ phase as reported on Figure 5. We observe as expected that the response is very close for the lower ITZ thickness $(25 \mu \mathrm{m})$, while significant discrepancies appear for the greater one $(250 \mu \mathrm{m})$. As a consequence and as already mentioned above, the ITZ should be properly characterized for modelling accurately the behavior of mortars, whereas for concrete viewed as a mix of a mortar matrix and aggregates 


\subsection{Local analyses}

We present in this section some statistical results regarding the distribution of the stresses and strains within the material. Figure 14 presents the probability distribution functions (PDF) of the longitudinal average stress (left) and strain (right) in the aggregates and matrix subvolumes for the interface thicknesses of 1 (top) and 250 (bottom) $\mu \mathrm{m}$, and at $0.38,24.5$ and 300 days. The matrix subvolumes are obtained by dividing the matrix with a voxel-type procedure. Here the dimensions of a voxel are chosen as 1/20 of the edge dimension of the initial box (i.e. 8000 subvolumes are expected).

We observe that the stress in the aggregates is slightly more dispersed than in the matrix subvolumes, in particular in the case of the smaller interface thickness. This is the contrary for the strain, which appears much more dispersed in the matrix. This dispersion tends to reduce and increase for the average stress and strain, respectively, for higher thickness. Moreover, in all cases the PDF are significantly more dispersed at later ages. These results indicate that the presence of interfaces affects notably the mean stress in both matrix and aggregate phases (as can be seen on Figure 11), but also their dispersion. Besides, the relatively high dispersion, especially in the matrix phase, proves that important stress and strain concentrations arise locally, meaning that 
microcracking is likely to occur in particular in the matrix and ITZ, whose strength is in general weaker than in the aggregates.

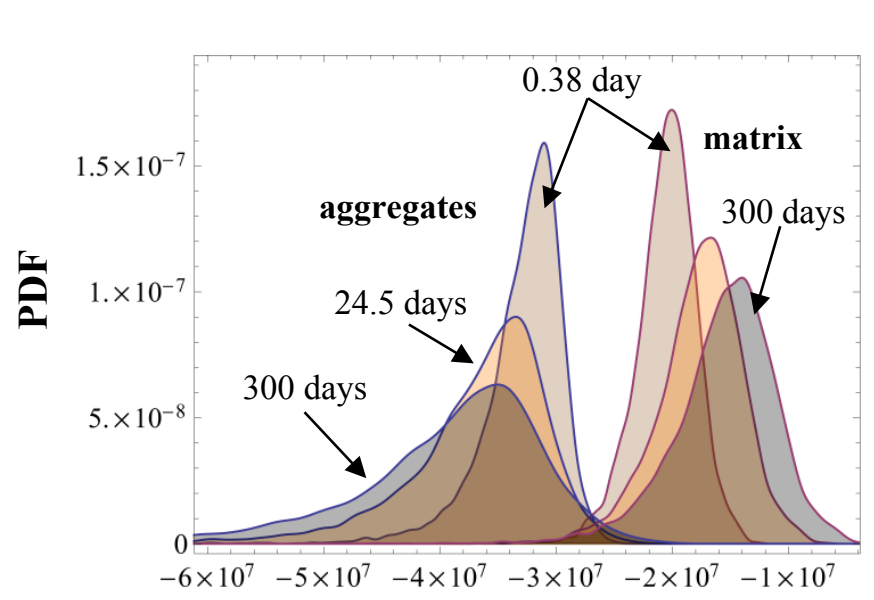

Stress (Pa)

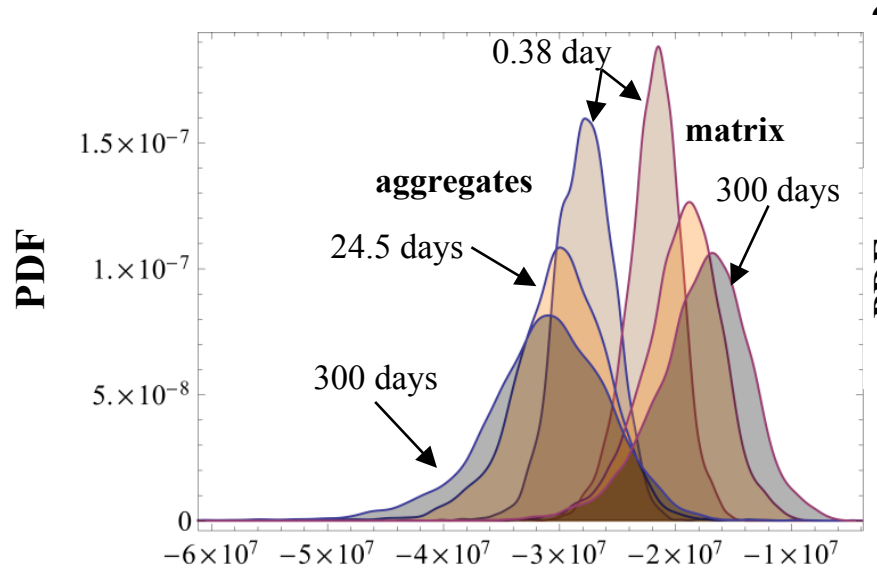

Stress $(\mathbf{P a})$

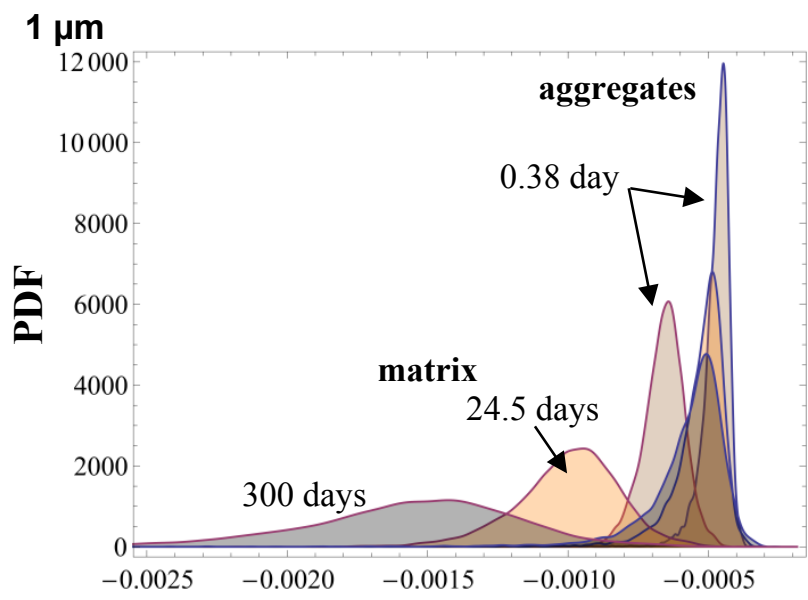

$250 \mu \mathrm{m}$

Strain (-)

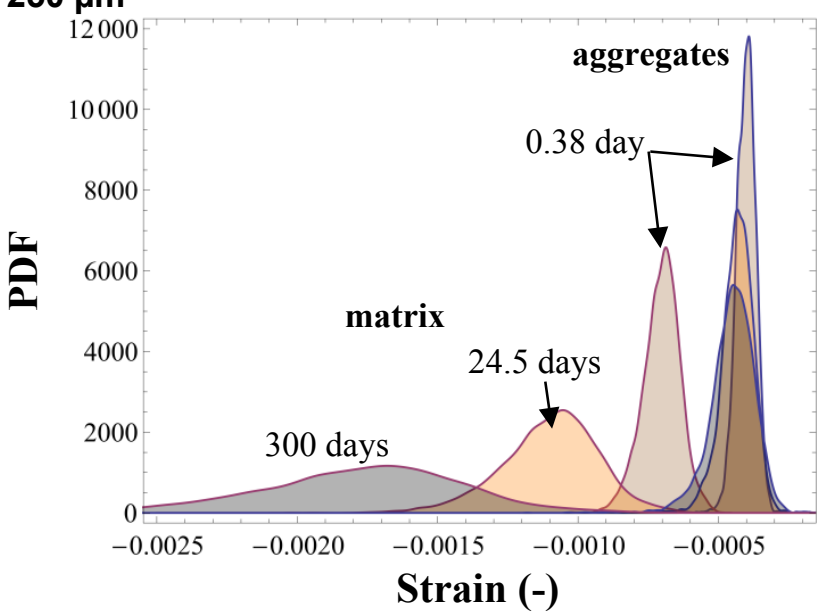

Figure 14. FE Probability Distribution Function for the average longitudinal stress (left) and strain (right) in both matrix subvolumes and aggregates for the $1 \mu \mathrm{m}$ (top) and $250 \mu \mathrm{m}$ (bottom) interface thickness and at $0.38,24.5$ and 300 days.

\section{Extension to linear thermoviscoelasticity}

We extend in this subsection the formulation presented in section 2 to the case of linear thermoviscoelasticity. The effects of temperature have generally to be introduced in the model through different aspects. One relates to the viscoelastic behavior of the matrix phase, which is known to be strongly affected by temperature (see e.g. [58-60]. A simple approach to account for this effect is the time shift (or reduced time) method which consists in replacing the time variable $t$ by an equivalent time variable $t_{e}(t)$ accounting for temperature variations and defined as (see e.g. [59]): 


$$
t_{e}(t)=\int_{0}^{t} \exp \left[-\frac{Q}{R}\left(\frac{1}{T(\tau)}-\frac{1}{T_{0}}\right)\right] d \tau
$$

where $Q$ is an activation energy, $R$ is the ideal gas constant, $T_{0}$ is a reference temperature and $T$ is the current temperature. In the following applications, the ratio $Q / R$ is set to $6200 \mathrm{~K}$, which is close to values found in the literature for concrete materials (see e.g. [40,60]).

Another crucial aspect to consider regarding temperature effects is the dilatation of the material phases. It is indeed well known that for heterogeneous materials exhibiting phases with (in general) different coefficients of thermal expansion (CTE), internal stresses and strains may develop even with homogeneous temperature variations due to differential dilation effects. Here the CTE of aggregates $\alpha_{g}$ and matrix $\alpha_{m}$ are assumed to be isotropic, constant and equal to $1.1 \times 10^{-5}$ and $1.6 \times$ $10^{-5} \mathrm{~K}^{-1}$, respectively, corresponding to average values for sand and cement paste from $[61,62]$. The hypothesis of constant values seems reasonable in a moderate range of temperature variations as considered in this study. Note that in this case and taking into account the equivalent time $t_{e}(t)$ expressed above, the thermoviscoelastic behavior of the matrix may be formulated as:

$$
\boldsymbol{\sigma}^{m}(t)=3 \int_{0^{-}}^{t_{e}} k^{m}\left(t_{e}-\tau\right) \frac{d\left(\epsilon^{m}-\alpha^{m} \Delta T\right)}{d \tau} d \tau \mathbf{1}+2 \int_{0^{-}}^{t_{e}} \mu^{m}\left(t_{e}-\tau\right) \frac{d \boldsymbol{e}^{m}}{d \tau} d \tau
$$

In the LC space the behavior takes the generic following form:

$$
\tilde{\boldsymbol{\sigma}}_{m}(s)=3 \tilde{k}^{m}(s)\left(\tilde{\epsilon}_{m}-\alpha^{m} \Delta T\right) \mathbf{1}+2 \tilde{\mu}^{m}(s) \tilde{\boldsymbol{e}}_{m}
$$

The CTE of the ITZ phase $\alpha_{c}$ is estimated from the matrix one with the same method than for its mechanical properties, i.e. by describing it as a composite constituted of the matrix material with additional porosity. It is well known that for such 2-phase material the effective CTE in the case of isotropic linear elasticity $\alpha_{\text {eff }}^{(2)}$ is obtained exactly by means of the Levin formula, providing the knowledge of the effective compressibility modulus $k_{\text {eff }}^{(2)}[63]$ :

$$
\alpha_{\text {eff }}^{(2)}=\frac{\alpha_{1}\left(1 / k_{\text {eff }}^{(2)}-1 / k_{2}\right)-\alpha_{2}\left(1 / k_{\text {eff }}^{(2)}-1 / k_{1}\right)}{1 / k_{1}-1 / k_{2}}
$$

In the case where the phase 2 is porosity, then $k_{2}=0$ and consequently $\alpha_{\text {eff }}^{(2)}=\alpha_{1}$. It implies for the ITZ that $\alpha_{c}=\alpha^{m}$.

The next step of the modelling is to calculate the effective CTE $\alpha_{e f f}$ of the 3-phase material depicted on Figure 3 left, i.e. composed of spherical aggregates surrounded by an ITZ layer and immersed in the matrix. We make use here of the developments of [64] to derive $\alpha_{\text {eff }}$ in the case 
of thin interfaces with elastic mechanical parameters much lower than those of the inclusions, leading to the following explicit expression (in the LC space):

$$
\frac{\tilde{\alpha}_{e f f}-\phi_{g} \alpha_{g}-\phi_{m} \alpha_{m}-\frac{\phi_{g} \tilde{\gamma}}{R_{i}}}{\frac{1}{\tilde{k}_{e f f}}-\frac{\phi_{g}}{\tilde{k}_{g}}-\frac{\phi_{m}}{\tilde{k}_{m}}-\frac{3}{\tilde{k}_{n} R_{i}}}=\frac{\alpha_{g}-\alpha_{m}+\frac{\tilde{\gamma}}{R_{i}}}{\frac{1}{\tilde{k}_{g}}-\frac{1}{\tilde{k}_{m}}+\frac{3}{\tilde{k}_{n} R_{i}}}
$$

where the subscripts $m, c$ and $g$ designate again the matrix, interphase and aggregate, respectively; $\phi_{i}$ is the volume fraction of phase $i ; \tilde{k}_{n}$ is defined in Eqs. (9). Finally, $\tilde{\gamma}$ is the normal thermoelastic parameter of the interface defined by (see section 2.2 for the details of the notation):

$$
[\boldsymbol{u}] \cdot \boldsymbol{n}=\tilde{\gamma} \Delta T
$$

with $\Delta T$ the homogeneous increment of temperature. The parameter $\tilde{\gamma}$ defines then the response of the interfaces in terms of normal displacement jump when subjected to a temperature variation. Again, we refer here on the results derived by [64] to express $\tilde{\gamma}$ as a function of the thermoelastic properties of both interphase and aggregates, and the interface thickness $h$, in the form:

$$
\tilde{\gamma}=\frac{\tilde{\alpha}_{c}\left(1+\tilde{v}_{c}\right)-2 \alpha_{g} \tilde{v}_{c}}{1-\tilde{v}_{c}} h
$$

Note that Eq. (17) constitutes an extension of the Levin formula to the particular 3-phase case of the composite sphere assemblage represented on Figure 3 left. Figure 15 left depicts the time evolution of $\alpha_{e f f}$ as obtained with this formulation for different ITZ thicknesses. We observe that $\alpha_{e f f}$ evolves slightly (about $2.5 \%$ ) in the range 0-50 days, and that it is also moderately affected by the ITZ thickness (approximately $6 \%$ of difference between 1 and $250 \mu \mathrm{m}$ ). Figure 15 right shows the evolution of the effective CTE $\alpha_{e f f}^{(G S C S)}$ obtained with the GSCS applied to the 3-phase representation of the material, i.e. considering explicitly the ITZ (see Figure 3 left), and expressed in [65] (and also in [66] in a different form) as:

$$
\alpha_{e f f}^{(G S C S)}=\frac{\tilde{k}_{m}}{k_{e f f}^{(G S C S)}} \alpha_{m}+\frac{\left(3 \tilde{k}_{m}+4 \tilde{\mu}_{m}\right)\left(\tilde{k}_{e f f}^{(2)} \tilde{\alpha}_{e f f}^{(2)}-\tilde{k}_{m} \alpha_{m}\right)\left(1-\phi_{m}\right)}{k_{e f f}^{(G S C S)}\left(4 \tilde{\mu}_{m}+3 \tilde{k}_{e f f}^{(2)} \xi_{m}+3 \tilde{k}_{m}\left(1-\phi_{m}\right)\right)}
$$

where $k_{e f f}^{(G S C S)}$ is the effective bulk modulus of the 3-phase material, $\tilde{k}_{e f f}^{(2)}$ and $\tilde{\alpha}_{e f f}^{(2)}$ denote the effective bulk modulus and CTE, respectively, of the 2-phase sphere assemblage consisting of the aggregates surrounded by the ITZ. We observe that the influence of the ITZ thickness is much weaker for $\alpha_{e f f}^{(G S C)}$ than for $\alpha_{e f f}$; this result agrees with Figure 12 ones showing the effects of ITZ on the macroscopic creep strains. Note that from the numerical viewpoint, the resulting 
thermoviscoelastic behaviors of both interface and matrix have been implemented via the code generator MFront, and the general thermomechanics formulation of Cast3M has been applied.

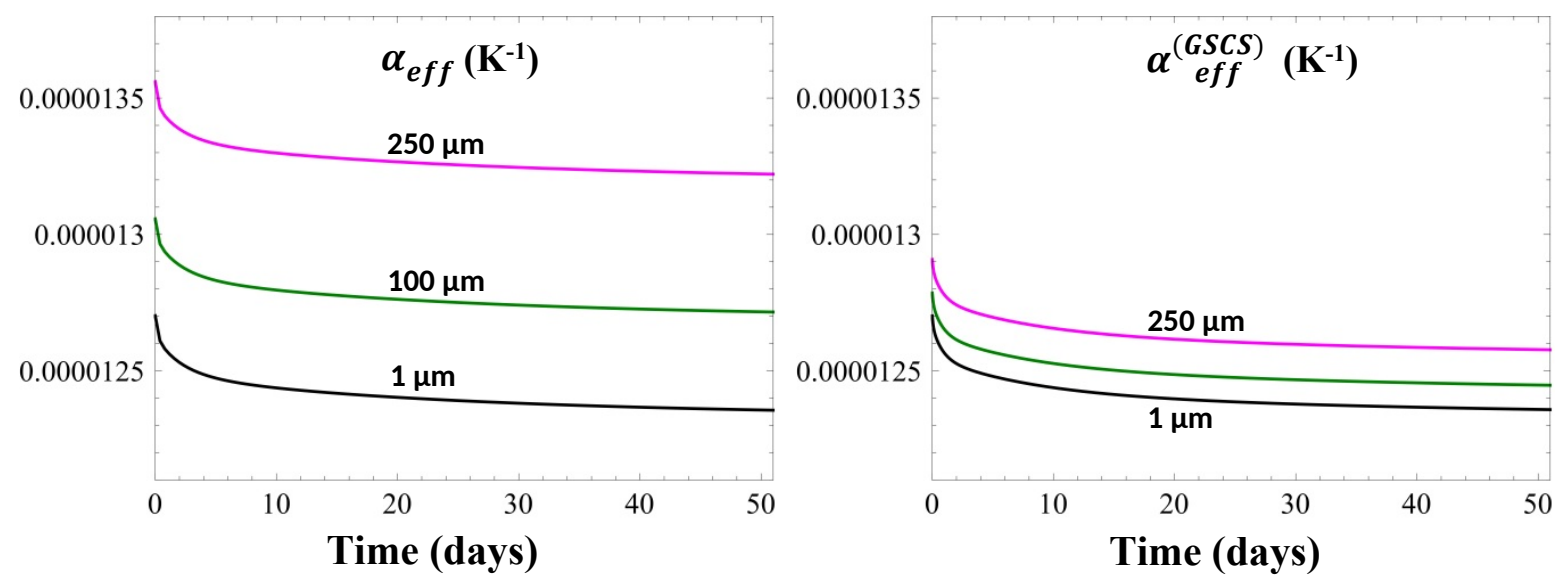

Figure 15. Evolution of the macroscopic CTE $\alpha_{e f f}$ according to Eq. (17) (left) and $\alpha_{\text {eff }}^{(G S C S)}$ according to GSCS with 3 phases defined in Eq. 20 (right) for interface thicknesses of 1, 100 and $250 \mu \mathrm{m}$.

We present in the following some illustrative simulation results obtained with the mesostructure with isotropic aggregates, where the only external loading is a temperature increase of $40^{\circ} \mathrm{C}$ (no macroscopic stresses or strains are imposed). The simulations are performed with homogeneous stresses SUBC. The analytical estimations are derived with the replacement procedure and the GSCS with 2 phases. Figure 16 shows the evolution during the first 50 days of the isotropic macroscopic strains obtained numerically (symbols) and analytically (lines) for the interface thicknesses of 1,100 and $250 \mu \mathrm{m}$. We observe that the magnitude of these strains is only moderately affected by the ITZ thickness, as could be expected from Figure 15; indeed, in this particular case the strain tensor is simply given by $\boldsymbol{\varepsilon}(t)=\alpha_{e f f}(t) \Delta T \mathbf{1}$, which means that the time evolution of $\boldsymbol{\varepsilon}$ follow the ones of $\alpha_{e f f}$ whatever the ITZ thickness. Specifically, the strain difference between the lower and higher thickness value is no more than $5 \%$. Moreover, the time evolution of the macroscopic strains is also limited to about $2.5 \%$ at 50 days, as for $\alpha_{e f f}(t)$. The comparison between FE numerical and analytical results shows a relatively good agreement especially for $h=$ $250 \mu \mathrm{m}$ (maximum discrepancy of less than $0.4 \%$ at 50 days), and small differences appear for lower thicknesses (about $1.5 \%$ at 50 days for $h=1 \mu \mathrm{m}$ ).

Figure 17 presents the evolution of the average stresses in aggregate and matrix phases obtained numerically and analytically for the 3 considered interface thicknesses. As the volume fraction of the inclusion phase is $50 \%$ and no macroscopic stresses are applied to the specimen, the average stresses in both phases are opposite (note that this is also a consequence of the interface behavior, modelled here with the LSM and implying that there is no jump of the normal stress vector across 


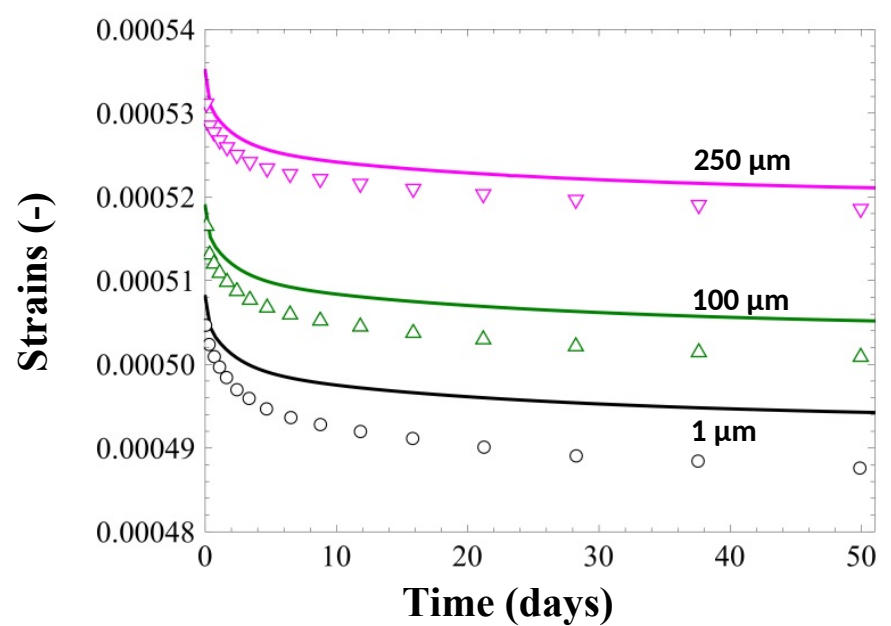

Figure 16. Macroscopic strains due to a temperature increase of $40^{\circ} \mathrm{C}$ obtained numerically (symbols) and analytically (lines) for interface thicknesses of 1 (black), 100 (green) and 250 (pink) $\mu \mathrm{m}$.

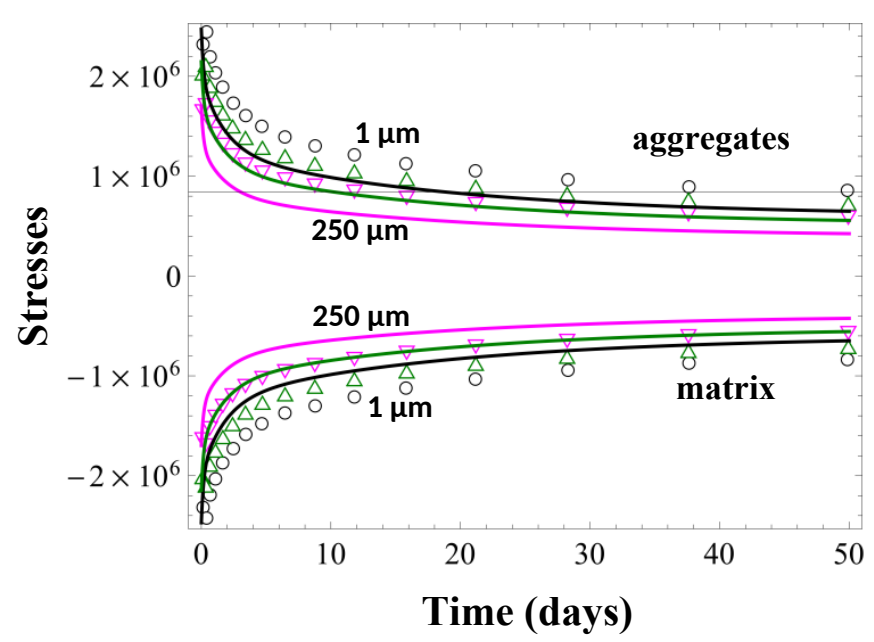

Figure 17. Average stresses in the matrix and aggregate phase due to a temperature increase of $40^{\circ} \mathrm{C}$ obtained numerically (symbols) and analytically (lines) for interface thicknesses of 1 (black), 100 (green) and 250 (pink) $\mu \mathrm{m}$. 

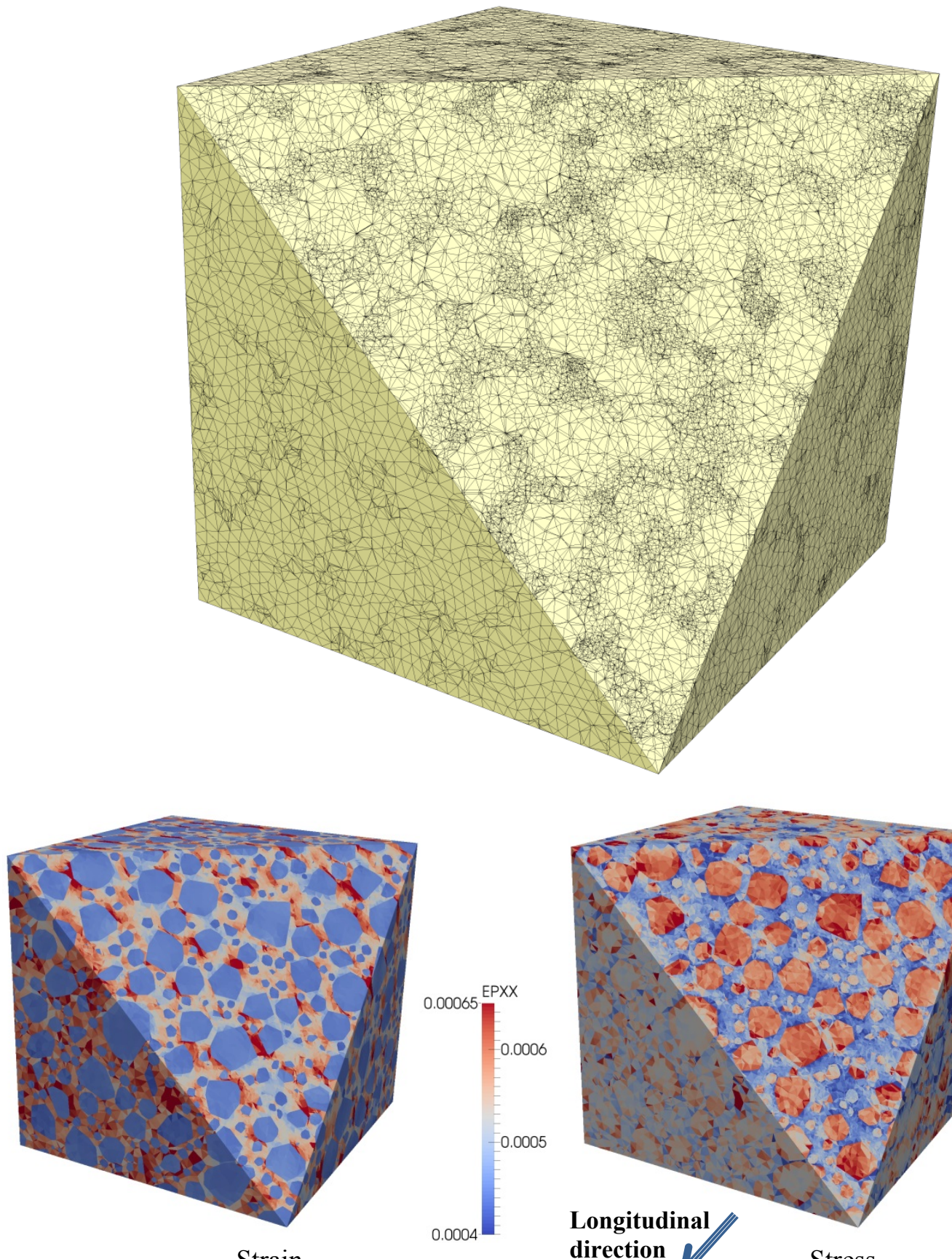

Strain

Figure 18. Mesh of a $50 \%$ mesostructure used in the simulations (top). Distribution of longitudinal strain (bottom left) and stress (bottom right, Pa) at 1 day in the case of ITZ thickness of $100 \mu \mathrm{m}$ after a temperature increase of $40^{\circ} \mathrm{C}$ (bottom).

Finally, Figure 18 illustrates the distribution of longitudinal strain (bottom, left) and stress (bottom, right) within the mesostructure at 1 day in the case of the ITZ thickness of $100 \mu \mathrm{m}$. The mesh used in the corresponding simulations is represented in Figure 18 top. We indeed observe that 
aggregate phase is globally in tension and matrix one in compression, although the total strains are greater in the matrix. We also note the (quasi-)zero normal stress on the front left surface of the mesostructure perpendicular to the longitudinal direction, resulting from the homogeneous zero stress boundary conditions.

To conclude this section, we consider again from the numerical-analytical confrontation that the analytical model developed is globally able to correctly reproduce the main features of the material behavior. As already mentioned, the observed relatively moderate discrepancies are attributed for a large part to the fact that the analytical estimations provide approximate solutions by nature, and that they are based on a representation with spherical aggregates while the numerical samples are generated with polyhedrons. More generally, further investigations regarding this latter aspect, including taking into account in the analytical estimations the ITZ surrounding non-spherical aggregates, could improve the model prediction capacities.

\section{Conclusion}

We have analyzed analytically and numerically in this paper the thermoviscoelastic behavior of concrete and mortar, considered at mesoscale as heterogeneous materials composed of a matrix in which are embedded aggregates surrounded by an ITZ. FE simulations have been performed on 3D samples, some of them exhibiting more than 4600 polyhedral aggregates randomly distributed in a box with a total volume fraction of $50 \%$. Both matrix and ITZ behaviors have been considered as linear thermoviscoelastic and the aggregates as elastic. Specific FE interface elements have been introduced to model the ITZ, whose properties have been estimated from the matrix ones. Analytical estimations of the material response have been provided especially by applying a procedure for replacing the composite inclusions (i.e., initial aggregates considered as spherical + ITZ) by equivalent homogeneous particles, and then by using the GSCS in the Laplace-Carson space. In such space the ITZ are governed by the Linear Spring Model. The overall and intra-phase responses in the numerical specimens have been investigated when subjected to classical creep loading, and compared to the analytical estimations. The results obtained show that typical ITZ thicknesses for concrete of 20-25 $\mu \mathrm{m}$ do not affect significantly its behavior, contrary to the case of mortars whose response is comparatively notably influenced. As expected, increasing the ITZ thickness leads globally to a rise of the creep strains magnitude, i.e. the material becomes more compliant. Moreover, it implies a reduction in magnitude of the stresses in the aggregates and an increase of both stresses and strains in the matrix phase. A significant dispersion has been observed in the average creep stress per aggregate and per matrix subvolumes, indicating potentially important local stress concentrations. Higher ITZ thicknesses tend to slightly reduce this 
dispersion. The effects of the aggregate shape (i.e. flat or elongated polyhedrons with aspect ratio of 4) have also been analyzed on the macroscopic response and prove to be limited but nonnegligible, whatever the interface thickness. Finally, an application of the modelling procedure to temperature increase is presented; in particular, the coefficient of thermal expansion is estimated for the composite material including the ITZ. Again, the comparison of analytical and numerical results shows a fairly good agreement, indicating that the model is able to relatively correctly reproduce the thermoviscoelastic behavior of concrete as represented in this study.

\section{Acknowledgments}

The CEA MATIX project, EDF and MACENA project are gratefully acknowledged for financial support.

\section{References}

[1] M.P. Lutz, P.J.. Monteiro, R.W. Zimmerman, Inhomogeneous interfacial transition zone model for the bulk modulus of mortar, Cement and Concrete Research. 27 (1997) 1113-1122.

[2] K.L. Scrivener, A.K. Crumbie, P. Laugesen, The interfacial transition zone (ITZ) between cement paste and aggregate in concrete, Interface Science. 12 (2004) 411-421.

[3] F. Lavergne, K. Sab, J. Sanahuja, M. Bornert, C. Toulemonde, Investigation of the effect of aggregates' morphology on concrete creep properties by numerical simulations, Cement and Concrete Research. 71 (2015) 14-28. doi:10.1016/j.cemconres.2015.01.003.

[4] J.P. Ollivier, J.C. Maso, B. Bourdette, Interfacial transition zone in concrete, Advanced Cement Based Materials. 2 (1995) 30-38. doi:10.1016/1065-7355(95)90037-3.

[5] Z. Sun, E.J. Garboczi, S.P. Shah, Modeling the elastic properties of concrete composites: Experiment, differential effective medium theory, and numerical simulation, Cement and Concrete Composites. 29 (2007) 22-38.

[6] F. Bernard, S. Kamali-Bernard, Numerical study of ITZ contribution on mechanical behavior and diffusivity of mortars, Computational Materials Science. 102 (2015) 250-257.

[7] S. Caré, E. Hervé, Application of a n-phase model to the diffusion coefficient of chloride in mortar, Transport in Porous Media. 56 (2004) 119-135.

[8] J. Zheng, H.S. Wong, N.R. Buenfeld, Assessing the influence of ITZ on the steady-state chloride diffusivity of concrete using a numerical model, Cement and Concrete Research. 39 (2009) 805-813. doi:10.1016/j.cemconres.2009.06.002.

[9] W. Dridi, Analysis of effective diffusivity of cement based materials by multi-scale modelling, Mater Struct. 46 (2013) 313-326. doi:10.1617/s11527-012-9903-5.

[10] B. Bary, N. Leterrier, E. Deville, P. Le Bescop, Coupled chemo-transport-mechanical modelling and numerical simulation of external sulfate attack in mortar, Cement and Concrete Composites. 49 (2014) 70-83. doi:10.1016/j.cemconcomp.2013.12.010.

[11] Z. Hashin, Thermoelastic properties of particulate composites with imperfect interface, Journal of the Mechanics and Physics of Solids. 39 (1991) 745-762.

[12] H.L. Duan, X. Yi, Z.P. Huang, J. Wang, A unified scheme for prediction of effective moduli of multiphase composites with interface effects. Part I: Theoretical framework, Mechanics of Materials. 39 (2007) 81-93. doi:10.1016/j.mechmat.2006.02.009.

[13] J. Wang, H.L. Duan, Z. Zhang, Z.P. Huang, An anti-interpenetration model and connections between interphase and interface models in particle-reinforced composites, International Journal of Mechanical Sciences. 47 (2005) 701-718. doi:10.1016/j.ijmecsci.2004.12.014. 
[14] H. Tan, Y. Huang, C. Liu, P.H. Geubelle, The Mori-Tanaka method for composite materials with nonlinear interface debonding, International Journal of Plasticity. 21 (2005) 1890-1918.

[15] Y.J. Wei, L. Anand, Grain-boundary sliding and separation in polycrystalline metals: application to nanocrystalline fcc metals, Journal of the Mechanics and Physics of Solids. 52 (2004) 2587-2616.

[16] F. Grondin, M. Matallah, How to consider the Interfacial Transition Zones in the finite element modelling of concrete?, Cement and Concrete Research. 58 (2014) 67-75.

[17] A. Caballero, C.M. López, I. Carol, 3D meso-structural analysis of concrete specimens under uniaxial tension, Computer Methods in Applied Mechanics and Engineering. 195 (2006) 7182-7195. doi:10.1016/j.cma.2005.05.052.

[18] S.-M. Kim, R.K. Abu Al-Rub, Meso-scale computational modeling of the plastic-damage response of cementitious composites, Cement and Concrete Research. 41 (2011) 339-358.

[19] H.-K. Man, J.G.M. van Mier, Influence of particle density on 3D size effects in the fracture of (numerical) concrete, Mech. Mater. 40 (2008) 470-486.

[20] D. Asahina, E.N. Landis, J.E. Bolander, Modeling of phase interfaces during pre-critical crack growth in concrete, Cement and Concrete Composites. 33 (2011) 966-977.

[21] B. Šavija, J. Pacheco, E. Schlangen, Lattice modeling of chloride diffusion in sound and cracked concrete, Cement and Concrete Composites. 42 (2013) 30-40.

[22] J. Yvonnet, H. Le Quang, Q.C. He, An XFEM/level set approach to modelling surface/interface effects and to computing the size-dependent effective properties of nanocomposites, Computational Mechanics. 42 (2008) 119-131.

[23] Q.-Z. Zhu, S.-T. Gu, J. Yvonnet, J.-F. Shao, Q.-C. He, Three-dimensional numerical modelling by XFEM of spring-layer imperfect curved interfaces with applications to linearly elastic composite materials, International Journal for Numerical Methods in Engineering. 88 (2011) 307-328. doi:10.1002/nme.3175.

[24] G. Alfano, E. Sacco, Combining interface damage and friction in a cohesive-zone model, Int. J. Numer. Meth. Engng. 68 (2006) 542-582. doi:10.1002/nme.1728.

[25] X.F. Wang, Z.J. Yang, J.R. Yates, A.P. Jivkov, C. Zhang, Monte Carlo simulations of mesoscale fracture modelling of concrete with random aggregates and pores, Construction and Building Materials. 75 (2015) 35-45. doi:10.1016/j.conbuildmat.2014.09.069.

[26] T. Wu, P. Wriggers, Multiscale diffusion-thermal-mechanical cohesive zone model for concrete, Comput Mech. (2015) 1-18. doi:10.1007/s00466-015-1149-y.

[27] B. Bary, M. Ben Haha, E. Adam, P. Montarnal, Numerical and analytical effective elastic properties of degraded cement pastes, Cement and Concrete Research. 39 (2009) 902-912. doi:10.1016/j.cemconres.2009.06.012.

[28] T. de Larrard, B. Bary, E. Adam, F. Kloss, Influence of aggregate shapes on drying and carbonation phenomena in 3D concrete numerical samples, Computational Materials Science. 72 (2013) 1-14. doi:10.1016/ j.commatsci. 2013.01.039.

[29] T.T.H. Nguyen, B. Bary, T. de Larrard, Coupled carbonation-rust formation-damage modeling and simulation of steel corrosion in 3D mesoscale reinforced concrete, Cement and Concrete Research. 74 (2015) 95-107. doi:10.1016/j.cemconres.2015.04.008.

[30] B. Bary, C. Bourcier, T. Helfer, Numerical analysis of concrete creep on mesoscopic 3D specimens, in: Mechanics and Physics of Creep, Shrinkage, and Durability of Concrete and Concrete Structures, Vienne, Austria, 2015.

[31] C.F. Dunant, B. Bary, A.B. Giorla, C. Péniguel, J. Sanahuja, C. Toulemonde, A.-B. Tran, F. Willot, J. Yvonnet, A critical comparison of several numerical methods for computing effective properties of highly heterogeneous materials, Advances in Engineering Software. 58 (2013) 1-12. doi:10.1016/j.advengsoft.2012.12.002.

[32] J. Mandel, Cours de mécanique des milieux continus, Gauthier-Villars, 1966.

[33] S. Beurthey, A. Zaoui, Structural morphology and relaxation spectra of viscoelastic heterogeneous materials, European Journal of Mechanics - A/Solids. 19 (2000) 1-16.

[34] N. Laws, R. McLaughlin, Self-consistent estimates for the viscoelastic creep compliances of composite materials, Proc. R. Soc. Lond. A. 359 (1978) 251-273.

[35] L.C. Brinson, W.S. Lin, Comparison of micromechanics methods for effective properties of multiphase viscoelastic composites, Composite Structures. 41 (1998) 353-367. 
[36] C. Donolato, Analytical and numerical inversion of the Laplace-Carson transform by a differential method, Computer Physics Communications. 145 (2002) 298-309.

[37] M. Lévesque, M.D. Gilchrist, N. Bouleau, K. Derrien, D. Baptiste, Numerical inversion of the Laplace-Carson transform applied to homogenization of randomly reinforced linear viscoelastic media, Comput Mech. 40 (2006) 771-789. doi:10.1007/s00466-006-0138-6.

[38] R.A. Schapery, Approximate methods of transform inversion for viscoelastic stress analysis, in: Proc. 4th US Nat'l Cong. Appl. Mech, 1962: pp. 1075-1085.

[39] A. Rekik, R. Brenner, Optimization of the collocation inversion method for the linear viscoelastic homogenization, Mechanics Research Communications. 38 (2011) 305-308. doi:10.1016/j.mechrescom.2011.04.003.

[40] M.-Q. Thai, B. Bary, Q.-C. He, A homogenization-enriched viscodamage model for cement-based material creep, Engineering Fracture Mechanics. 126 (2014) 54-72.

[41] H.L. Duan, X. Yi, Z.P. Huang, J. Wang, A unified scheme for prediction of effective moduli of multiphase composites with interface effects: Part II-Application and scaling laws, Mechanics of Materials. 39 (2007) 94-103. doi:10.1016/j.mechmat.2006.02.010.

[42] Z. Hashin, Thin interphase/imperfect interface in elasticity with application to coated fiber composites, Journal of the Mechanics and Physics of Solids. 50 (2002) 2509-2537.

[43] S.-T. Gu, J.-T. Liu, Q.-C. He, Size-dependent effective elastic moduli of particulate composites with interfacial displacement and traction discontinuities, International Journal of Solids and Structures. 51 (2014) 2283-2296. doi:10.1016/j.ijsolstr.2014.02.033.

[44] T. Mori, K. Tanaka, Average stress in matrix and average elastic energy of materials with misfitting inclusions, Acta Metallurgica. 21 (1973) 571-574.

[45] R. Christensen, K. Lo, Solutions for effective shear properties in three phase sphere and cylinder models, Journal of the Mechanics and Physics of Solids. 27 (1979) 315-330.

[46] Z. Hashin, Thermoelastic properties of fiber composites with imperfect interface, Mechanics of Materials. 8 (1990) 333-348. doi:10.1016/0167-6636(90)90051-G.

[47] C. Bourcier, W. Dridi, L. Chomat, E. Laucoin, B. Bary, E. Adam, Combs: open source python library for RVE generation. Application to microscale diffusion simulations in cementitious materials, in: Paris, 2013.

[48] S. Thomas, Y. Lu, E.J. Garboczi, Improved model for three-dimensional virtual concrete: anm model, Journal of Computing in Civil Engineering. 30 (2016) 04015027.

[49] W. Ladaoui, Etude expérimentale du comportement Thermo-Hydro-Mécanique à long terme des BHP destinés aux ouvrages de stockage des déchets radioactifs, Université de Toulouse, Université Toulouse III-Paul Sabatier, 2010. http://thesesups.ups-tlse.fr/1160/ (accessed December 1, 2013).

[50] C.M. Neubauer, H.M. Jennings, E.J. Garboczi, A three-phase model of the elastic and shrinkage properties of mortar, Advanced Cement Based Materials. 4 (1996) 6-20.

[51] T. Helfer, B. Michel, J.-M. Proix, M. Salvo, J. Sercombe, M. Casella, Introducing the open-source mfront code generator: Application to mechanical behaviours and material knowledge management within the PLEIADES fuel element modelling platform, Computers \& Mathematics with Applications. 70 (2015) 994-1023.

[52] T. Kanit, S. Forest, I. Galliet, V. Mounoury, D. Jeulin, Determination of the size of the representative volume element for random composites: statistical and numerical approach, Int. J. Solids Struct. 40 (2003) 3647-3679. doi:10.1016/S0020-7683(03)00143-4.

[53] M. Salmi, F. Auslender, M. Bornert, M. Fogli, Apparent and effective mechanical properties of linear matrix-inclusion random composites: Improved bounds for the effective behavior, International Journal of Solids and Structures. 49 (2012) 1195-1211.

[54] K. Sab, On the homogenization and the simulation of random materials, European Journal of Mechanics. A. Solids. 11 (1992) 585-607.

[55] S. Pecullan, L.V. Gibiansky, S. Torquato, Scale effects on the elastic behavior of periodic and hierarchical two-dimensional composites, Journal of the Mechanics and Physics of Solids. 47 (1999) $1509-1542$.

[56] Z. Hashin, P. Monteiro, An inverse method to determine the elastic properties of the interphase between the aggregate and the cement paste, Cement and Concrete Research. 32 (2002) 1291-1300. 
[57] Y. Huang, K.X. Hu, X. Wei, A. Chandra, A generalized self-consistent mechanics method for composite materials with multiphase inclusions, Journal of the Mechanics and Physics of Solids. 42 (1994) 491-504.

[58] S. Arthanari, C.W. Yu, Creep of concrete under uniaxial and biaxial stresses at elevated temperatures, Mag. Concr. Res. 19 (1967) 149-156.

[59] Z.P. Bazant, G. Cusatis, L. Cedolin, Temperature effect on concrete creep modeled by microprestresssolidification theory, J. Eng. Mech. 130 (2004) 691-699.

[60] W. Ladaoui, T. Vidal, A. Sellier, X. Bourbon, Effect of a temperature change from 20 to $50^{\circ} \mathrm{C}$ on the basic creep of HPC and HPFRC, Materials and Structures. 44 (2011) 1629-1639.

[61] C.R. Cruz, M. Gillen, Thermal expansion of Portland cement paste, mortar and concrete at high temperatures, Fire Mater. 4 (1980) 66-70. doi:10.1002/fam.810040203.

[62] M. Wyrzykowski, P. Lura, Controlling the coefficient of thermal expansion of cementitious materials - A new application for superabsorbent polymers, Cement and Concrete Composites. 35 (2013) 4958. doi:10.1016/j.cemconcomp.2012.08.010.

[63] V.M. Levin, On the coefficients of thermal expansion of heterogeneous materials, Mechanics of Solids. 2 (1967) 58-61.

[64] H.L. Duan, B.L. Karihaloo, Thermo-elastic properties of heterogeneous materials with imperfect interfaces: Generalized Levin's formula and Hill's connections, Journal of the Mechanics and Physics of Solids. 55 (2007) 1036-1052. doi:10.1016/j.jmps.2006.10.006.

[65] B. Bary, S. Bejaoui, Assessment of diffusive and mechanical properties of hardened cement pastes using a multi-coated sphere assemblage model, Cement and Concrete Research. 36 (2006) 245-258. doi:10.1016/j.cemconres.2005.07.007.

[66] E. Hervé, Thermal and thermoelastic behaviour of multiply coated inclusion-reinforced composites, International Journal of Solids and Structures. 39 (2002) 1041-1058. 
- 3D specimens are generated with thermoviscoelastic matrix and dispersed particles

- Thermoviscoelastic interfaces between particles and matrix are introduced

- FE results are successfully compared to various upscaling schemes estimations

- Impact of interface thickness is significant for mortar and negligible for concrete

- Effects of flattened particle shapes on overall and local behavior are moderate 